\title{
Familie und Familienschutz im Wandel - zur Entwicklung des Familienbegriffs im öffentlichen Recht
}

\author{
Friederike Wapler"
}

A. Ausgangspunkt: kontroverse Diskussionen im Parlamentarischen Rat..... 58

I. Eheliche Familie oder „Ehe und Familie“? ....................... 58

II. Der Streit um das Elternrecht ....... 60

III. Patriarchales Eherecht und die Gleichberechtigung der Geschlechter............................. 62

B. Gleichberechtigung von Mann und Frau im Eherecht: Zum Verhältnis von Art. 6 Abs. 1 GG zu Art. 3 Abs. 2 GG. .63

C. Der verfassungsrechtliche Begriff der Familie....

I. Vorrang der Ehe oder Gleichberechtigung aller Familienformen?....... 67

II. Familie als Lebensgemeinschaft aus Eltern und Kindern: ein zu enger Begriff?

III. Die nichteheliche Familie............

1. Die Rechtsstellung des nichtehelichen Vaters
2. Nichteheliche Elternschaft und medizinische Reproduktion...... 72

a. Der Zugang zu medizinischen Reproduktionstechniken.....

b. Elternschaft nach künstlicher Befruchtung............... 73

c. Multiple Elternschaft....... 76

D. Der Wunsch nach einem Familienleben: die Rechtsstellung des leiblichen, aber nicht rechtlichen Vaters.......... 77

E. Gleichgeschlechtliche Familien ........ 79

F. Das Kind als Familienmitglied........ 82 I. Das Recht des Kindes auf Entwicklung zu einer selbstbestimmten Person .............................. 82

II. Das Recht des Kindes auf Schutz.... 83

III. Der Schutz der familiären Bindungen des Kindes......................... 85

G. Öffentliche Familienförderung....... 86

Als der Parlamentarische Rat in den Jahren 1948 und 1949 tagte, um das künftige Grundgesetz der Bundesrepublik Deutschland auszuarbeiten, entbrannte zwischen den Delegierten heftiger Streit um die Familie. Dies war insofern erstaunlich, als in den ersten Entwürfen zum Grundgesetz gar keine Bestimmung zur Familie vorgesehen gewesen war. ${ }^{1}$ Erst in der achten Sitzung des Grundsatzausschusses des Parlamentarischen Rates am 7.10.1948 schlug die Abgeordnete Weber einen Artikel zu Ehe und Familie vor und griff damit eine zentrale Forderung der beiden christlichen Kirchen auf. ${ }^{2}$ Die dadurch ausgelösten Auseinandersetzungen verdienen nähere Betrachtung, da sie Grundpositionen widerspiegeln, die bis heute in die familienpolitische und rechtliche Diskussion hineinwirken.

* PD Dr. Friederike Wapler ist Inhaberin der Entlastungsprofessur für Öffentliches Recht an der GoetheUniversität Frankfurt am Main.

1 Vgl. Bericht über den Verfassungskonvent auf Herrenchiemsee vom 10.-23.8.1948, in: Deutscher Bundestag/Bundesarchiv (Hrsg.), Der Parlamentarische Rat 1948-1949. Akten und Protokolle, Bd. 2, Boppard a. Rh. 1981, S. 504 (580-582).

2 H. Weber, in: Der Parlamentarische Rat (Fn. 1), Bd. 5/II, 1993, S. 218. 


\section{A. Ausgangspunkt: kontroverse Diskussionen im Parlamentarischen Rat}

\section{Eheliche Familie oder „Ehe und Familie“?}

Streitig war zum einen, welche Art der sozialen Gemeinschaft unter den besonderen Schutz der Verfassung gestellt werden sollte. Für die Abgeordneten der christlich geprägten Fraktionen von CDU und Zentrum war klar, dass die Verfassung nur eheliche Lebensverhältnisse besonders schützen sollte, nicht aber nichteheliches $\mathrm{Zu}$ sammenleben, „das Konkubinat“, wie von Mangoldt es ausdrückte. ${ }^{3}$ Der Vorschlag der CDU-Fraktion für einen Artikel zum Schutz von Ehe und Familie sah dementsprechend vor, den Familienschutz auf „die Ehe als rechtmäßige Form der dauernden Lebensgemeinschaft von Mann und Frau und die aus ihr wachsende Familie“ zu begrenzen. ${ }^{4}$ Allerdings hielt man eine Schutzvorschrift zugunsten von Ehe und Familie fraktionsübergreifend für eine rein programmatische Erklärung, die keine subjektiven Rechte verleihe. ${ }^{5}$ Mit dieser Begründung verkürzte der Redaktionsausschuss des Parlamentarischen Rates den Artikel auf die Formulierung „Ehe und Familie stehen unter dem besonderen Schutz des Staates“, die nach einigen weiteren Diskussionen schließlich Eingang in das Grundgesetz fand (heute Art. 6 Abs. 1 GG). ${ }^{6}$ Damit blieb sprachlich offen, ob zwischen Ehe und Familie ein notwendiger Zusammenhang bestehen sollte oder nicht.

Die Verfechter eines an die Ehe gekoppelten Familienschutzes lehnten sich konsequenterweise auch gegen eine rechtliche Gleichstellung der nichtehelichen - im damaligen Sprachgebrauch und auch im Wortlaut des Grundgesetzes „unehelichen“Kinder auf: Nach dem seinerzeit geltenden $\ 1598$ Abs. 2 BGB war das nichteheliche Kind lediglich mit seiner Mutter verwandt, was für die Vertreter der konservativen Fraktionen bedeutete, dass es ohne Familie war. Seine Lebenssituation war aus ihrer Sicht mit der eines ehelichen Kindes nicht vergleichbar und eine rechtliche Gleich-

3 H. von Mangoldt, in: Der Parlamentarische Rat (Fn. 1), Bd. 5/II, 1993, S. 827.

4 A. Süsterhenn, in: Der Parlamentarische Rat (Fn. 1), Bd. 5/II, 1993, S. 642.

5 Vgl. L. Bergsträsser (SPD), in: Der Parlamentarische Rat (Fn. 1), Bd. 5/II, 1993, S. 643; siehe auch die Diskussionen in der 29. Sitzung des Grundsatzausschusses, ebd., S. 808 ff., 826. In diesem Sinne auch die frühe Kommentierung des Art. 6 Abs. 1 GG bei H. von Mangoldt, Das Bonner Grundgesetz, Berlin 1953, S. 38.

6 Siehe Art. 7a Abs. 1 im „Entwurf des Grundgesetzes in der vom Allgemeinen Redaktionsausschuss redigierten Fassung“ (Dezember 1948), in: Der Parlamentarische Rat (Fn. 1), Bd. 7, 1995, S. 138. Die Formulierung wurde vom Grundsatzausschuss in seiner 32. Sitzung am 11.1.1948 nicht übernommen, aber vom Hauptausschuss in seiner 43. Sitzung am 18.1.1949 auf Antrag der SPD beschlossen, vgl. Abänderungsvorschläge des Ausschusses für Grundsatzfragen, in: Der Parlamentarische Rat (Fn. 1), Bd. 5/II, 1993, S. 935 f. und 957 f.; Protokoll der 43. Sitzung des Hauptausschusses ebd., Bd. 14/II, 2009, S. 1346. 
stellung daher unmöglich. ${ }^{7}$ Das nichteheliche Kind konnte nach dieser Auffassung allenfalls die gleiche soziale Förderung verlangen wie das eheliche.

Demgegenüber beharrte der FDP-Abgeordnete Heuss auf einer klaren rechtlichen Gleichstellung nichtehelicher und ehelicher Kinder im Grundgesetz. Zur Begründung führte er nicht, wie man aus heutiger Sicht vermuten könnte, die individuellen Freiheitsrechte an, sondern den infolge des Krieges eklatanten „Frauenüberhang“. ${ }^{8}$ Dieser führe zu der tragischen Situation, dass sich ledige Frauen Kinder wünschten, ohne dass aber eine Eheschließung in Frage käme. Dem sekundierte die SPD-Delegierte Selbert mit dem Hinweis, dass auch viele verheiratete Frauen an den Kriegsfolgen litten, insbesondere dann, wenn ihre Männer im Krieg unbekannt verschollen waren. Ihre Ehen konnten bis auf Weiteres nicht aufgelöst oder geschieden werden. Wollten diese Frauen neue Partnerschaften eingehen und Kinder bekommen, konnten sie dies folglich nur in nichtehelichen Arrangements verwirklichen. ${ }^{9}$

Von konservativer Seite stellte sich Weber gegen eine derart pragmatische Sicht der Dinge: Es gebe kein Recht auf ein uneheliches Kind, da die natürliche Ordnung der Familie dem entgegenstehe. ${ }^{10}$ Immer wieder argumentierte die christlich-konservative Seite damit, dass das Familienrecht des BGB Ausdruck einer natürlichen Ordnung sei, die der Verfassungsgeber als vorgefunden zu akzeptieren habe, und an der individuelle Ansprüche auf Gleichberechtigung notwendig zerschellen müssten. ${ }^{11}$

Die traditionelle rechtliche Schlechterstellung des nichtehelichen Kindes wurde auf diese Weise zu einer naturgegebenen Tatsache umgedeutet. Die SPD konterte dies mit dem Hinweis, dass die gegenwärtige Familienvorstellung historisch bedingt sowie sozial und rechtlich konstruiert sei:

„Die Rechtsfolgen, die wir an die Tatsache einer Blutsverwandtschaft knüpfen, sind nicht von Natur aus - nicht ,physei, sondern ,thesei ${ }^{*}$, sind Produkte der Rechtsordnung; und diese schaffen die Menschen. Die Familie ist, so seltsam Ihnen das klingen mag, als Institution, an die sich bestimmte Rechte und Pflichten knüpfen, ein Produkt der Rechtsordnung, und je nach den Rechtsordnungen, die in den einzelnen Epochen und in den einzelnen Gegenden gelten,

7 Vgl. A. Süsterhenn, in: Der Parlamentarische Rat (Fn. 1), Bd. 5/II, 1993, S. 644: „Ein eheliches Kind lebt in der Familie unter der Obhut von Vater und Mutter. Das uneheliche Kind lebt nicht in einer Familie, sondern steht in einem einseitigen Verhältnis zur Mutter. Solche Verschiedenheiten kann man durch keine Verfassungsbestimmung aus der Welt schaffen. Man kann höchstens sagen: Das uneheliche Kind soll die gleiche gesellschaftliche Förderung erhalten wie ein eheliches. "Siehe auch $H$. Weber, a.a.O., Bd. 14/I, 2009, S. 605: „Eine Gleichstellung ist niemals möglich, weil das Kind nicht in die Familie hineingeboren wird. Das Kind ist schuldlos, aber tragisch getroffen."

8 Vgl. Th. Heuss, in: Der Parlamentarische Rat (Fn. 1), Bd. 5/II, 1993, S. 644 und ebd., S. 809.

9 E. Selbert, in: Der Parlamentarische Rat (Fn. 1), Bd. 14/II, 2009, S. 1340 f.

10 H. Weber, in: Der Parlamentarische Rat (Fn. 1), Bd. 5/II, 1993, S. 811; in diesem Sinne auch H. Wessel, in: Der Parlamentarische Rat (Fn. 1), Bd. 14/I, 2009, S. 600.

11 Vgl. A. Süsterhenn, in: Der Parlamentarische Rat (Fn. 1), Bd. 14/I, 2009, S. 606 f.: „[...] die natürlich gegebene und aus der Natur erwachsende Ordnung ist auch uns als Gesetzgeber bindend vorgeschrieben $[\ldots]$." 
ist die Familie etwas vollkommen anderes, und es knüpfen sich an gleiche biologische und soziologische Tatbestände verschiedene Rechtsfolgen. "12

Folgerichtig forderte die SPD, die Verwandtschaft des nichtehelichen Kindes mit seinem Vater in der Verfassung ausdrücklich festzuschreiben. ${ }^{13}$ Dieser Vorschlag konnte sich jedoch ebenso wenig durchsetzen wie der konservative Versuch, den Schutz der Familie an den der Ehe zu koppeln. Im Ergebnis lehnte man den Passus zum nichtehelichen Kind an die entsprechende Vorschrift der Weimarer Reichsverfassung an. ${ }^{14}$ Die darin enthaltene Formulierung, „durch die Gesetzgebung die gleichen Bedingungen" für das nichteheliche wie das eheliche Kind zu schaffen, kann als allgemeiner sozialstaatlicher Förderauftrag wie als echtes Gleichheitsrecht gelesen werden. Eine eindeutige Entscheidung für die eine oder andere Lesart lässt sich den Protokollen des Parlamentarischen Rates nicht entnehmen.

\section{Der Streit um das Elternrecht}

Nicht nur die Frage nach den schützenswerten Lebensverhältnissen war im Parlamentarischen Rat streitig, sondern auch das Elternrecht. Hier entzündete sich der Konflikt an den religiösen Erziehungsrechten in der Schule: Während etliche Abgeordnete mit dem Rückhalt der christlichen Kirchen für ein weitreichendes elterliches Bestimmungsrecht über die konfessionelle Ausrichtung der Schulen plädierten, sprachen sich andere für ein konfessionell neutrales Gemeinschaftsschulsystem aus. ${ }^{15}$ Jenseits dieses schulbezogenen Streits, der bis zum Ende der Beratungen nicht ausgeräumt werden konnte, stand das private Erziehungsrecht der Eltern in der Familie jedoch nicht ernsthaft in Frage. Zwar stellten die einen es als „natürliches“ oder "gottgegebenes" Recht auf ein naturrechtliches bzw. theologisches Fundament, ${ }^{16}$ während der Liberale Heuss es schlicht als „Selbstverständlichkeit“ und „Banalität“

12 C. Schmid, in: Der Parlamentarische Rat (Fn. 1), Bd. 14/I, 2009, S. 604.

13 F. Nadig, in: Der Parlamentarische Rat (Fn. 1), Bd. 14/I, 2009, S. 599: „Notwendig wäre [...] eine Festlegung dahingehend, daß das uneheliche Kind auch mit seinem natürlichen Vater als verwandt gilt. Damit wäre überhaupt erst eine wirkliche Gleichstellung des unehelichen Kindes gegeben."; dies. ebd., S. 602: „Es ist doch wohl das natürlichste Recht, daß das Kind mit seinem Vater, selbst wenn er gesetzlich nur ,Erzeuger' genannt wird, verwandt ist."

14 Art. 121 WRV: „Den unehelichen Kindern sind durch die Gesetzgebung die gleichen Bedingungen für ihre leibliche, seelische und gesellschaftliche Entwicklung zu schaffen wie den ehelichen." Die Formulierung wurde dahingehend ergänzt, dass die gleichen Bedingungen auch für die „gesellschaftliche Stellung " des Kindes gelten sollen.

15 Vgl. die äußerst kontroversen Diskussionen auf der 29. Sitzung des Grundsatzausschusses des Parlamentarischen Rates, in: Der Parlamentarische Rat (Fn. 1), Bd. 5/II, 1993, S. 814-835, und auf der 21. Sitzung des Hauptausschusses, in: Der Parlamentarische Rat (Fn. 1), Bd. 14/1, 2009, S. 613-617.

16 Die Bezeichnung als „natürliches Menschenrecht“ stammt von Weber, vgl. Der Parlamentarische Rat (Fn. 1), Bd. 14/1, 2009, S. 613; siehe auch die Bezeichnung als „natürliches Recht“ bei Wessel, ebd., S. 614, und bei Bergsträsser, in: Der Parlamentarische Rat (Fn. 1), Bd. 5/II, 1993, S. 813. Zur Gottgegebenheit des Elternrechts siehe Weber, in: Der Parlamentarische Rat (Fn.1), Bd. 14/II, 2009, S. 1349 f.; Süsterhenn, ebd., S. 1490. 
bezeichnete. ${ }^{17}$ Im Ergebnis war man sich dennoch einig darin, sich klar von einer staatlich gelenkten Erziehung, wie man sie in der NS-Zeit erlebt hatte und wie sie sich in der Sowjetischen Besatzungszone bereits für die Zukunft abzeichnete, abzugrenzen. ${ }^{18}$ Am Ende fand man für das allgemeine Erziehungsrecht der Eltern eine Formulierung, die Art.120 WRV nachempfunden war - mit dem durchaus nicht unerheblichen Detail, dass das Elternrecht dort als „oberste Pflicht und natürliches Recht“ bezeichnet worden war, während in Art. 6 Abs. 2 S. 2 GG von dem „obersten Recht und der zuvörderst ihnen zukommenden Pflicht" gesprochen wird. ${ }^{19}$ Indem man das Recht der Eltern ihrer Pflicht sprachlich vorordnete, betonte man den abwehrrechtlichen Charakter des Elternrechts gegen erzieherische und kontrollierende Eingriffe des Staates.

Keine Aussprache fand in den Ausschüssen des Parlamentarischen Rates zum Wächteramt des Staates (Art. 6 Abs. 2 S. 2 GG) statt. Der Passus wurde erst wenige Tage vor der Verabschiedung des Grundgesetzes ohne Diskussion und einstimmig in den Verfassungstext aufgenommen, nachdem der SPD-Abgeordnete Zinn ihn auf einer interfraktionellen Besprechung als gemeinsamen Antrag der SPD- und der FDPFraktion eingebracht hatte. ${ }^{20}$ Kaum thematisiert wurde auch die Rechtsstellung des Kindes in Familie und politischer Gemeinschaft. Offenkundig herrschte ein Konsens darüber, dass die Rechte des Kindes im Regelfall durch die Eltern bzw. andere rechtlich vertretungsberechtigte Personen wahrgenommen wurden. In diese Richtung geht jedenfalls die einzige Äußerung eines Abgeordneten, in der von einem Recht des Kindes die Rede ist:

„Ich glaube, wir sind uns alle darin einig, daß die Eltern, und nur sie, das ausschließliche Recht haben, zu bestimmen, in welchen Formen und unter welchen Bedingungen ibre Kinder erzogen und herangebildet werden sollen. Zweitens dürften wir uns alle darin einig sein, daß den Kindern auch nur dieses eine ausschließliche Recht zusteht, und da sie unmündig sind, müssen die Eltern dieses Recht für sie ausüben. "21

In dieser Äußerung manifestiert sich die traditionelle Vorstellung, dass Rechte nur ausüben kann, wer einen autonomen Willen bilden kann, also mündig ist. Wer - wie Kindern in der zitierten Äußerung allgemein unterstellt wird - nicht mündig ist, muss

17 Vgl. Heuss, in: Der Parlamentarische Rat (Fn. 1), Bd. 5/II, 1993, S. 809, und ebd., Bd. 14/1, 2009, S. 617 .

18 Zur Abwehrhaltung gegenüber dem Nationalsozialismus siehe Der Parlamentarische Rat (Fn. 1), Bd. 14/1, 2009, S. 823 f.; zu dem Bestreben, sich „vom Osten [zu] distanzieren“, ebd., S. 814.

19 Art. 120 WRV: „Die Erziehung des Nachwuchses zur leiblichen, seelischen und gesellschaftlichen Tüchtigkeit ist oberste Pflicht und natürliches Recht der Eltern, über deren Betätigung die staatliche Gemeinschaft wacht."

20 Vgl. Interfraktionelle Besprechung v. 3.5.1949, in: Der Parlamentarische Rat (Fn. 1), Bd. 11, 1997, S. 226 f. und Sitzung des interfraktionellen Unterausschusses (4.5.1949), ebd., S. 268. Zur Abstimmung in der 57. Sitzung des Hauptausschusses siehe Der Parlamentarische Rat (Fn. 1), Bd. 14/II, 2009, S. 1815; zur Abstimmung im Plenum siehe 9. Sitzung des Plenums v. 6.5.1949, in: Der Parlamentarische Rat (Fn. 1), Bd. 9, 1996, S. 451.

21 J. Brockmann (Zentrum), in: Der Parlamentarische Rat (Fn. 1), Bd. 14/II, 2009, S. 1360. 
in der Ausübung seiner Rechte vertreten werden. Im Verhältnis von Eltern und Kindern erhält dieses Vertretungsverhältnis noch dazu den Charakter eines Insichgeschäfts, da die Eltern, wenn sie das Recht des Kindes auf Erziehung ausüben, zugleich die Verpflichteten dieses Rechts sind. Für das Kind bleibt es in diesem Fall ohne Belang, ob seine Eltern lediglich von Verfassungs wegen zu seiner Erziehung verpflichtet werden oder ob ihm als Kehrseite der Pflicht ein Recht auf Erziehung zusteht. Dass die Grundrechte als subjektive Rechte eines jeden Individuums auch für die Rechtsstellung des Kindes eine gewisse Sprengkraft entfalten können, war den Abgeordneten im Parlamentarischen Rat offenkundig nicht bewusst und sollte auch bis in die 1960er Jahre hinein ein Randthema in der juristischen Diskussion bleiben.

\section{Patriarchales Eherecht und die Gleichberechtigung der Geschlechter}

Keineswegs unterschätzt wurde im Parlamentarischen Rat hingegen die revolutionäre Kraft, die der Satz „Männer und Frauen sind gleichberechtigt“ in Art. 3 Abs. 2 GG für die Rechtsverhältnisse in der Familie barg. Im Gegenteil konzentrierten sich die Bedenken gegen diese Formulierung im Wesentlichen darauf, dass erhebliche Teile des BGB-Familienrechts mit einem solchen formalen Gleichheitsversprechen offenkundig nicht zu vereinbaren waren. ${ }^{22}$ Für das Familienrecht, so die vorherrschende Überzeugung der christlich-konservativen Abgeordneten, sei formale Gleichheit der Geschlechter nicht zu erreichen. Die Gleichberechtigung der Frau müsse ihre Grenze dort finden, wo sie mit den „natürlichen Funktionen der Geschlechter" nicht vereinbar sei. ${ }^{23}$

Eine gewisse Differenzierung wegen des Geschlechts hielten auch die Befürworter der umfassenden formalen Gleichstellung für zulässig, etwa hinsichtlich der Regelungen zur Mutterschaft: „Gleichberechtigung“ bedeute nicht notwendig „Gleichmacherei“, erklärte beispielsweise eine der einflussreichsten Streiterinnen für die heutige Form des Art. 3 Abs. 2 GG, Selbert. ${ }^{24}$ Gleichzeitig betonte sie, dass die formale Gleichstellung der Geschlechter durchaus erhebliche Veränderungen im Familienrecht erforderlich machen würde. ${ }^{25}$ So geschah es denn auch, nachdem das Bundesverfassungsgericht im Jahr 1953 den bindenden Charakter des Art. 3 Abs. 2 GG festgestellt hatte. In der Folge musste vor allem das bürgerliche Eherecht weitreichend reformiert werden. Diese Veränderungen sind Gegenstand des folgenden Abschnitts (B), bevor anschließend (C bis G) die weitgehend von dem Ehebegriff abgelöste Entwicklung des Begriffs der Familie nachgezeichnet wird.

22 Vgl. Th. Dehler, in: Der Parlamentarische Rat (Fn. 1), Bd. 5/II, 1993, S. 748: „Dann ist das Bürgerliche Gesetzbuch verfassungswidrig."

23 A. Süsterhenn, in: Der Parlamentarische Rat (Fn. 1), Bd. 5/II, 1993, S. 643.

24 E. Selbert, in: Der Parlamentarische Rat (Fn. 1), Bd. 14/II, 2009, S. 1314; siehe auch C. Schmid, in: Der Parlamentarische Rat (Fn. 1), Bd. 14/I, 2009, S. 516.

25 E. Selbert, in: Der Parlamentarische Rat (Fn. 1), Bd. 14/I, 2009, S. 513. 


\section{B. Gleichberechtigung von Mann und Frau im Eherecht: Zum Verhältnis von Art. 6 Abs. 1 GG zu Art. 3 Abs. 2 GG}

Wenn auch der Verfassungstext des Art. 6 Abs. 1 GG im Ergebnis relativ offen formuliert ist, orientierte sich das Leitbild der Familie in der frühen Verfassungsinterpretation an der traditionellen Vorstellung der ehelichen Familie mit dem pater familias als Oberhaupt. ${ }^{26}$ Überwiegend bejahte man im Bereich der Familie naturrechtliche Einschränkungen des Gleichheitssatzes, verstand den Schutz der vermeintlich natürlichen Ordnung der Familie also als verfassungsrechtlich gebotene Einschränkung des Gleichberechtigungsanspruchs aus Art. 3 Abs. 2 GG. ${ }^{27}$

Demgegenüber stellte das Bundesverfassungsgericht schon im Jahr 1953 klar, dass Art. 3 Abs. 2 GG als bindendes Gleichheitsrecht grundsätzlich keine rechtlichen Differenzierungen aufgrund des Geschlechts mehr erlaubte. ${ }^{28}$ Auf dieser dogmatischen Grundlage beseitigte es unter anderem zwei Vorrechte des Ehemannes, die auch das Gleichberechtigungsgesetz von 1958 nicht vollständig abgeschafft hatte: die alleinige rechtliche Vertretung des Kindes und das Letztentscheidungsrecht in Konflikten um die Kindererziehung („Stichentscheid“). ${ }^{29}$ Beide Regelungen erklärte das Bundesverfassungsgericht für unvereinbar mit Art. 3 Abs. 2 GG, da sie die Ehefrau dem Ehemann in Angelegenheiten der Kindererziehung rechtlich unterordneten. ${ }^{30}$ In diesem Zusammenhang stellte das Bundesverfassungsgericht nicht nur klar, dass die rechtliche Gleichordnung der Mutter mit dem Vater „nicht geeignet [ist], die Selbstverwirklichung der Frau zu beeinträchtigen" ${ }^{31}$ sondern auch, dass das Familienrecht

26 Vgl. den Bezug auf die „Rechtsinstitute der Ehe und Familie [...] in ihren für die abendländische Kulturwelt kennzeichnenden Grundzügen [...]“ bei H. von Mangoldt, Grundrechte und Grundsatzfragen des Bonner Grundgesetzes, AöR 1949, S. 273 (283 f.); siehe auch ders, Grundgesetz (Fn. 5), S. 71; ders./F. Klein, Das Bonner Grundgesetz, 2. Aufl. Berlin 1975, S. 267.

27 Vgl. F. W. Bosch, Gleichberechtigung im Bereich der elterlichen Gewalt, SJZ 1950, Sp. 625 (641): „Die Leitungsgewalt des Mannes und Vaters ist ein bereits präjuristisch geltender Ordnungsgrundsatz; wenn die positive Rechtsordnung ihn anerkennt und beibehält, ist dies nicht nur verfassungsrechtlich in Ordnung, sondern auch deshalb - wie mir scheint - geboten, weil bei Widerstreit zwischen natürlicher Ordnung und positiver Rechtsordnung in jedem Falle die erstere vorgeht." Ähnlich auch $G$. Dürig, Art. 3 II GG - vom verfassungsrechtlichen Standpunkte her gesehen, FamRZ 1954, S. 2 (4). Zur Geschichte der Dogmatik des Art. 3 Abs. 2 GG ausführlich U. Sacksofsky, Das Grundrecht auf Gleichberechtigung, 2. Aufl. Baden-Baden 1996, S. 323 ff.; M. Wrase/A. Klose, Gleichheit unter dem Grundgesetz, in: L. Foljanty/U. Lembke (Hrsg.), Feministische Rechtswissenschaft. Ein Studienbuch, 2. Aufl. Baden-Baden 2012, S. 89-108; Th. Degener, Der Streit um Gleichheit und Differenz in der Bundesrepublik Deutschland seit 1945, in: U. Gerhardt (Hrsg.), Frauen in der Geschichte des Rechts, München 1997, S. 871 (881ff.).

28 BVerfGE 3, 225 (240); BVerfGE 10, 59 (73 f.).

29 Gleichberechtigungsgesetz vom 18.6.1957, BGBl. I 1957, S. 609. Darin \1628 Abs. 1 BGB: „Können sich die Eltern nicht einigen, so entscheidet der Vater; er hat auf die Auffassung der Mutter Rücksicht zu nehmen.“ \$ 1629 Abs. 1: „Die Vertretung des Kindes steht dem Vater zu; die Mutter vertritt das Kind, soweit sie die elterliche Gewalt allein ausübt oder ihr die Entscheidung nach $\mathbb{\$} 1628$ Abs. 2,3 [vormundschaftsgerichtlich, FW] übertragen ist."

30 BVerfGE 10, 59 (77 ff.).

31 BVerfGE 10, 59 (75). 
nunmehr von Verfassungs wegen einem neuen Leitbild unterlag: dem der einverständlichen Versorgung und Erziehung der Kinder durch beide Elternteile. ${ }^{32}$

Gleichzeitig aber trug das Bundesverfassungsgericht in den 1950er und 1960er Jahren durchaus selbst zu einer Naturalisierung der Geschlechterrollen bei, wie sie sich bereits in den Beratungen des Parlamentarischen Rates abgezeichnet hatte: Eine Differenzierung nach Geschlecht, so seine Rechtsprechung in dieser Zeit, sei immer dann zulässig, wenn „objektive biologische oder funktionale (arbeitsteilige) Unterschiede“ sie zwingend erforderten. ${ }^{33}$ Die „objektiven biologischen Unterschiede“ spielen vor allem auf rechtliche Regelungen im Zusammenhang mit der Schwangerschaft an, die bis heute als Sonderrecht für Frauen beispielsweise im Mutterschutzgesetz bestehen. Der Bezug auf „objektive funktionale (arbeitsteilige)“ Gründe aber öffnet ein weites Feld für Differenzierungen aus Rücksicht auf die traditionelle Rollenverteilung in der Ehe. So ist zu erklären, dass sich bis in das Jahr 1977 zwei Regelungen im BGB halten konnten, mit denen die Ehefrau dazu verpflichtet wurde, den Haushalt zu führen und zwar auch dann, wenn sie erwerbstätig war. ${ }^{34}$ Diese Bestimmungen bewertete das Bundesverfassungsgericht obiter dictum als verfassungsgemäß, wohl - wie das Gericht in den Entscheidungsgründen nur andeutet - weil die Beiträge des Mannes und der Frau zum Unterhalt zwar als andersartige, aber dennoch gleichwertige Beiträge zum gemeinschaftlichen Leben beschrieben werden, der Frau also für das, was sie in der Familie traditionellerweise leistete, die rechtliche Anerkennung nicht versagt blieb. ${ }^{35}$ Darin scheint die Vorstellung durch, die tradierte und im BGB rechtlich festgeschriebene Rollenverteilung in der Ehe habe einen objektiven, quasi natürlichen und für die Ausgestaltung des Rechts zwingenden Charakter. Das verfassungsrechtliche Leitbild der Familie geht damit bis in die 1970er Jahre hinein deutlich in die Richtung der traditionellen patriarchalen Ehe, in der die Aufgaben arbeitsteilig auf den Ernährer und die Hausfrau verteilt sind. Gleichberechtigung bedeutet in diesem Modell, die Gleichwertigkeit der unterschiedlichen Beiträge zu dieser ansonsten nicht veränderbaren Struktur rechtlich abzusichern. Daher sah das Bundesverfassungsgericht noch in den 1960er Jahren eine wesentliche Aufgabe des Gleichheitssatzes des Art. 3 Abs. 2 GG darin, „der rechtlichen Unterbewertung der Arbeit der Frau in

32 BVerfGE 10, 59 (79).

33 BVerfGE 3, 225 (242); BVerfGE 10, 59, (74); BVerfGE 15, 337 (343); BVerfGE 39, 169 (185); BVerfGE 48, 327 (337); BVerfGE 52, 369 (374).

$34 \$ 1356$ Abs. 1 BGB i.d.F. des Gleichberechtigungsgesetzes v. 1958: „Die Frau führt den Haushalt in eigener Verantwortung. Sie ist berechtigt, erwerbstätig zu sein, soweit dies mit ihren Pflichten in Ehe und Familie vereinbar ist. “; $\$ 1360$ Abs. 1 S. 2: „Die Frau erfüllt ihre Verpflichtung, durch Arbeit zum Unterhalt der Familie beizutragen, in der Regel durch die Führung des Haushaltes; zu einer Erwerbstätigkeit ist sie nur verpflichtet, soweit die Arbeitskraft des Mannes und die Einkünfte der Ehegatten zum Unterhalt nicht ausreichen [...].“

35 BVerfGE 10, 59 (57). Siehe auch BVerfGE 17, 1 (12). 
Haushalt und Familie ein Ende zu setzen und ihr eine gerechte Berücksichtigung zu sichern." 36

Dieses Bild von der Ehe änderte sich in der verfassungsgerichtlichen Rechtsprechung erst in den 1970er Jahren, als die tatsächlichen Verhältnisse sich wandelten und das Familienrecht in breiteren Bevölkerungskreisen als nicht mehr zeitgemäß empfunden wurde. Eine Ursache des veränderten Rechtsverständnisses war die zunehmende Berufstätigkeit verheirateter Frauen. Noch im Jahr 1963 hatte das Bundesverfassungsgericht geschlechtsdifferenzierende Regelungen damit gerechtfertigt, dass die Ehefrau sich von der gesetzlichen Pflicht zur Sorge für Haushalt und Kinder auch nicht durch Erwerbstätigkeit „loskaufen“ könne. ${ }^{37}$ Im Jahr 1975 revidierte es diese Haltung, da die Lebensverhältnisse sich gewandelt hätten und für Ehefrauen auch andere Gestaltungsmöglichkeiten als ein Dasein als Hausfrau möglich seien. ${ }^{38}$ Brachte die Ehefrau einen Teil ihres Unterhaltsbeitrags durch Erwerbstätigkeit ein, konnte sie sich dadurch nach dieser Rechtsprechung von ihren Haushaltspflichten ganz oder teilweise befreien. Das Bundesverfassungsgericht hielt es sogar für möglich, dass die Pflicht zur Haushaltsführung in diesen Fällen ganz oder teilweise auf den Mann überging. ${ }^{39}$ Dennoch erlaubte das Gericht dem Gesetzgeber, weiter typisierende Regelungen zu treffen, weil es die tatsächlichen Lebensverhältnisse und gesellschaftlichen Anschauungen zu jener Zeit in einer Übergangsphase sah. ${ }^{40} \mathrm{Zu}$ jener Zeit wurde gerade die sozialliberale Familienrechtsreform diskutiert, die im Jahr 1977 in Kraft trat. Neben umfangreichen anderen Änderungen, u.a. der Abschaffung des Schuldprinzips im Scheidungsrecht, wurden mit dieser Reform auch die geschlechtsspezifischen Rollenzuweisungen aus dem BGB entfernt. ${ }^{41}$

Der Bezug auf die „objektiven funktionalen Unterschiede“ findet sich in der Rechtsprechung des Bundesverfassungsgericht bis in die 1980er Jahre hinein. Jedoch betont das Gericht in den späteren Entscheidungen stärker, dass allein die traditionelle Prägung eines Lebensbereichs nicht ausreicht, um eine Ungleichbehandlung aufgrund des Geschlechts zu rechtfertigen. ${ }^{42}$ Während es also sprachlich auf der Linie seiner früheren Rechtsprechung bleibt, vollzieht es einen inhaltlichen Wandel: Statt wie früher darauf hinzuwirken, dass die Frau in ihrer traditionellen Rolle rechtlich bessergestellt wird, macht es etwa ab den 1980er Jahren zunehmend das emanzipatorische, gesellschaftliche Veränderungen aufgreifende und verstärkende Potential

41 Erstes Gesetz zur Reform des Ehe- und Familienrechts v. 14.6.1976, BGBl. I 1976, S. 1421, in Kraft ab dem 1.7.1977. Vgl. zu dieser Entwicklung Degener (Fn. 27), S. $882 \mathrm{f}$.

42 BVerfGE 57, 335 (342); BVerfGE 74, 163 (179f.); BVerfGE 84, 9 (17). 
der Regelung fruchtbar, indem es betont, dass die gesellschaftliche Wirklichkeit nicht so akzeptiert werden muss, wie man sie vorfindet. ${ }^{43}$ Doch erst im Jahr 1991 stellt es zum ersten Mal in Frage, ob „funktionale Unterschiede“ überhaupt als Rechtfertigung für Ungleichbehandlungen herangezogen werden können. ${ }^{44}$ Es lässt die Frage auf einer abstrakten Ebene offen, stellt jedoch klar:

„Eine Rechtfertigung der namensrechtlichen Ungleichbehandlung durch solche Unterschiede scheidet offensichtlich aus. Eine geringere Berufstätigkeit von Franen [...] ist nicht auf objektive funktionale Unterschiede zwischen den Geschlechtern zurückzuführen, sondern allenfalls auf eine traditionell typische Arbeitsteilung, die Art. 3 Abs. 2 GG gerade nicht verfestigen will $[\ldots] . " 45$

Die Argumentation ist ersichtlich inkonsequent: Wenn die traditionelle Arbeitsteilung der Geschlechter nicht mehr, wie in den 1950er und 1960er Jahren, als Ausdruck objektiver Unterschiede zwischen Mann und Frau akzeptiert wird, wenn man zudem postuliert, dass die Eheleute gleichberechtigt sind und über die Aufgabenteilung in der Familie frei entscheiden können, dann lässt dies nur den Schluss zu, dass es im Geschlechterverhältnis „objektive funktionale Unterschiede“ schlicht nicht gibt. Das Bundesverfassungsgericht hat diese Konsequenz nie ausdrücklich formuliert, das Kriterium der funktionalen Unterschiede zwischen den Geschlechtern nach 1991 jedoch stillschweigend aufgegeben. ${ }^{46}$ Die Gleichberechtigung von Mann und Frau in der Ehe steht seither ebenso wenig in Frage wie die Freiheit der Eheleute, ihr Familienleben nach ihren Wünschen auszugestalten. ${ }^{47}$ Auch das Verhältnis von Art. 6 Abs. 1 zu Art. 3 Abs. 2 GG ist damit geklärt: Der nach Art. 6 Abs. 1 GG gebotene Schutz von Ehe und Familie kann nicht dazu verwendet werden, mit dem Hinweis auf ein bestimmtes Leitbild der Familie - sei es traditionell begründet oder mit dem gesellschaftlichen Wandel neu entstanden - Ungleichbehandlungen aufgrund des Geschlechts zu rechtfertigen.

\section{Der verfassungsrechtliche Begriff der Familie}

Wie steht es aber mit dem Verhältnis der Ehe zur Familie? Das Bundesverfassungsgericht hat früh eine Linie eingeschlagen, die für eine weitgehende Entkoppelung beider Begriffe steht:

43 BVerfGE 84, 9 (17).

44 BVerfGE 105, 1 (18).

45 BVerfGE 105, 1 (18 f.).

46 Die letzte veröffentlichte Entscheidung, in der das Kriterium erwähnt wird, betrifft das Namensrecht des Kindes: BVerfG FamRZ 1991, 1161. Siehe zur weiteren Entwicklung der Dogmatik des Gleichheitssatzes aus Art. 3 Abs. 2 GG Sacksofsky (Fn. 27), S. 387; Degener (Fn. 27), S. 886 ff.

47 Vgl. statt vieler BVerfGE 103, 89 (101); BVerfGE 107, 27 (53); F. Brosius-Gersdorf, in: H. Dreier (Hrsg.), Grundgesetz-Kommentar, Bd. 1, 3. Aufl., Tübingen 2013, Art. 6 GG Rn. 66 ff.; D. CoesterWaltjen, in: I. von Münch/Ph. Kunig, Grundgesetz. Kommentar, Bd. 1, 6. Aufl. München 2012, Art. 6 GG Rn. 27 m.N. 
„Ehe ist auch für das Grundgesetz die Vereinigung eines Mannes und einer Frau zur grundsätzlich unauflöslichen Lebensgemeinschaft, und Familie ist die umfassende Gemeinschaft von Eltern und Kindern, in der Eltern vor allem Recht und Pflicht zur Pflege und Erziehung von Kindern erwachsen. "48

Dennoch wird die Frage, welcher Stellenwert der ehelichen Familie innerhalb der Vielfalt der tatsächlich gelebten Familienformen zusteht, in der Verfassungsrechtswissenschaft bis heute diskutiert.

\section{Vorrang der Ehe oder Gleichberechtigung aller Familienformen?}

Auf der Grundlage der oben zitierten Definition erkannte das Bundesverfassungsgericht schon früh nichteheliche Eltern-Kind-Beziehungen als Familie im Sinne des Art. 6 Abs. 1 GG an. ${ }^{49}$ Gleiches gilt für Adoptiv-, Stief- und Pflegekinderverhältnisse, die in ständiger Rechtsprechung ebenfalls unter den Familienschutz des Art. 6 Abs. 1 GG fallen. ${ }^{50}$ Die Ehelichkeit der elterlichen Verbindung spielt demnach für den verfassungsrechtlichen Familienbegriff ebenso wenig eine Rolle wie die leibliche Abstammung oder rechtliche Zuordnung des Kindes. ${ }^{51}$

Diese begriffliche Zuordnung sagt jedoch noch nichts über die inhaltliche Bewertung der unterschiedlichen Familienformen aus. Die aus den Beratungen des Parlamentarischen Rates geschilderte Vorstellung, die Ehe sei das einzig rechtmäßige Institut der Paargemeinschaft und Kindererziehung, zeichnet sich auch in der frühen Rechtsprechung des Bundesverfassungsgerichts ab. In den 1950er und 1960er Jahren konstruiert es ein verfassungsrechtliches Rangverhältnis zwischen der Ehe und anderen Formen der Familie:

„Die vom Verfassungsgeber vorausgesetzte ungünstige Ausgangsposition des unehelichen Kindes berubt gerade darauf, daß nach den in Art. 6 Abs. 1 GG verfassungsrechtlich garantierten Wertvorstellungen die Ehe die einzig legitime Form umfassender Lebensgemeinschaft zwischen Mann und Frau ist und die gesunde körperliche und seelische Entwicklung des Kindes grundsätzlich das Geborgensein in der nur in der Ehe verwirklichten vollständigen Familiengemeinschaft mit Vater und Mutter voraussetzt [...]. "52

Die Vorstellung, dass die eheliche Kleinfamilie der beste Ort sei, um Kinder aufzuziehen, findet sich bis heute in der staatsrechtlichen Literatur. ${ }^{53}$ Das Bundesverfas-

49 BVerfGE 8, 210 (215); BVerfGE 24, 119 (135); BVerfGE 25, 167 (196).

50 Nichteheliche Kinder: BVerfGE 8, 210 (215); BVerfGE 24, 119 (135); 25, 167 (196); BVerfGE 79, 256 (267); BVerfGE 106, 166 (176). Stief-, Adoptions- und Pflegekinder: BVerfGE 18, 97 (105 f.); BVerfGE 79, 256 (267).

51 Brosius-Gersdorf (Fn. 47), Art. 6 GG Rn. 44.

52 BVerfGE 25, 167 (196); BVerfGE 56, 363, Ziff. 65; BVerfGE 76, 1 (51). Siehe aus der Literatur von Mangoldt, Grundgesetz (Fn. 5), S. 71 und ders./Klein, Grundgesetz (Fn. 26), S. 267.

53 Siehe P. Badura, in: Th. Maunz/G. Dürig (Hrsg.), Grundgesetz-Kommentar, 25. EL April 2012, Art. 6 GG Rn. 93; U. Di Fabio, Der Schutz von Ehe und Familie: Verfassungsentscheidung für eine vitale Gesellschaft, NJW 2003, S. 993 (994); C. Seiler, Grundzüge eines öffentlichen Familienrechts, Tübingen 2008, S. $36 \mathrm{f}$. 
sungsgericht hat dieses Verständnis des Art. 6 Abs. 1 GG hingegen schon in den späten 1980er Jahren aufgegeben. Der letzte Bereich, in dem es bis heute die einfachrechtliche Einschätzung, die Ehe trage den Belangen des Kindes am besten Rechnung, als verfassungsgemäß verteidigt, betrifft die künstliche Befruchtung. Dass die Krankenkassen Leistungen der medizinischen Reproduktion nur bei Ehepaaren übernehmen ( $\$ 27$ a Abs. 1 Nr. 3 SGB V), hat es im Jahr 2007 für verfassungsgemäß - nicht allerdings für verfassungsrechtlich geboten - erklärt. ${ }^{54}$ Doch auch für diese abgeschwächte Billigung eines einfachrechtlichen Ehevorrangs findet sich verfassungsdogmatisch keine Rechtfertigung. Das Bindewort „und“ zwischen „Ehe“ und „Familie“ spricht für eine Gleichordnung beider Formen der sozialen Gemeinschaft. Betrachtet man den Entstehungsprozess der Vorschrift, so lässt sich ebenfalls kaum sagen, dass die Befürworter eines Ehevorrangs sich durchgesetzt hätten. Zwar waren die Stimmen im Parlamentarischen Rat, die eine besondere Bevorzugung der ehelichen Familiengemeinschaft forderten, recht stark und prägten nach 1949 vor allem in der Gestalt von Mangoldts die Interpretation des Art. 6 Abs. 1 GG. Im Ergebnis jedoch fand nicht der ehezentrierte Vorschlag der christlich geprägten Fraktionen eine Mehrheit, sondern die heutige, gegenüber der Weimarer Reichsverfassung deutlich neutralere Formulierung. Auch der Verfechter einer Koppelung von Ehe und Familie, von Mangoldt, sieht diese Bedeutung des Wortlauts, wenn er in seiner ersten Kommentierung des neuen Grundgesetzes im Jahr 1953 schreibt, im Parlamentarischen Rat scheine „eine gewisse Scheu vor einer entschiedenen Ablehnung der außerehelichen Formen der Lebensgemeinschaft von Mann und Frau bestanden zu haben. " ${ }^{55}$ Systematisch spricht die mittlerweile allgemein anerkannte abwehrrechtliche Funktion des Art. 6 Abs. 1 GG - Freiheit der Familiengründung und der Ausgestaltung des Familienlebens - ebenfalls dafür, eine grundsätzliche Gleichwertigkeit aller Familienformen anzunehmen, zumal sich empirisch nicht nachweisen lässt, dass das Aufwachsen in einer Ehe für Kinder signifikant besser ist als ein Leben in einer nichtehelichen Familienkonstellation. Der verfassungsrechtliche Begriff der Familie umfasst folglich alle Formen des Zusammenlebens von Eltern und Kindern, und alle diese Konstellationen sind grundsätzlich als gleichberechtigt zu behandeln. ${ }^{56}$

\section{Familie als Lebensgemeinschaft aus Eltern und Kindern: ein zu enger Begriff?}

Während mit der Ehe eine Lebensgemeinschaft geschützt ist, die auch kinderlos bleiben kann, setzt der verfassungsrechtliche Familienbegriff ein Eltern-Kind-Verhältnis voraus. Keinen verfassungsrechtlichen Schutz aus Art. 6 Abs. 1 GG genießen dem- 
zufolge nichteheliche Lebensgemeinschaften ohne Kinder. ${ }^{57} \mathrm{Ihr}$ Zusammenleben fällt jedoch unter die allgemeine Handlungsfreiheit nach Art. 2 Abs. 1 GG; auch sind begrenzte Gleichbehandlungsansprüche nichtehelicher Lebensgemeinschaften im Vergleich zur Ehe aus Art. 3 Abs. 1 GG anerkannt. ${ }^{58}$

Die enge Definition der Familie als Zwei-Generationen-Gemeinschaft kollidiert des Weiteren mit einem weiten Alltagsverständnis, das neben der Kleinfamilie aus Vater, Mutter und Kindern auch Geschwisterverhältnisse sowie die Beziehungen zu Großeltern, Tanten, Cousinen, Schwägern etc. als „Familie“ begreift. Das Bundesverfassungsgericht hat den Familienbegriff des Art. 6 Abs. 1 GG bislang auf die Kleinfamilie aus Eltern und Kindern beschränkt und eine Erweiterung auf Groß- und Mehrgenerationenfamilien abgelehnt. ${ }^{59}$ Anders die Rechtsprechung des Europäischen Gerichtshofs für Menschenrechte, der jedenfalls das Großeltern-Enkel-Verhältnis in den Begriff des Familienlebens gem. Art. 8 EMRK einbezieht. ${ }^{60}$ Diese Auffassung verdient Zustimmung: Der besondere Schutz der Familie bezieht sich auf Verantwortungsbeziehungen zwischen Erwachsenen und Kindern. Verantwortung und Solidarität zwischen den Generationen besteht vielfach über das Eltern-Kind-Verhältnis hinaus, etwa wenn Großeltern mit ihren Kindern und Enkeln unter einem Dach leben oder Tanten und Onkel sich an der Kinderbetreuung beteiligen. Es spricht darum einiges dafür, den Familienschutz des Art. 6 Abs. 1 GG jedenfalls auf solche verwandtschaftlichen Beziehungen zu erweitern, die von gegenseitiger Fürsorge und langfristiger Verantwortungsübernahme geprägt sind. ${ }^{61}$

\section{Die nichteheliche Familie}

Bereits sehr früh, im Jahr 1958, hat das Bundesverfassungsgericht die Lebensgemeinschaft einer Mutter mit ihrem nichtehelichen Kind als Familie anerkannt, die verfassungsrechtlichen Schutz genießt. ${ }^{62}$ Diese Anerkennung bezog sich zunächst jedoch nur auf die Konstellation der alleinerziehenden Mutter. Lebten Mann und Frau ohne förmliche Eheschließung mit oder ohne Kinder zusammen, so bereitete diese Verbindung als „Konkubinat“ oder „wilde Ehe“ nicht nur dem Familienrecht, sondern auch der Verfassungsinterpretation über viele Jahre erhebliche Schwierigkeiten.

57 BVerfGE 87, 234 (264); Brosius-Gersdorf(Fn. 47), Art. 6 GG Rn. 57; E. Schumann, Die nichteheliche Lebensgemeinschaft, in: Soergel (Hrsg.), Kommentar zum Bürgerlichen Gesetzbuch, Bd. 17/2, Stuttgart 2013, Rn. 23; dies., Die nichteheliche Familie, München 1998, S. 186 f.

58 Siehe den Überblick über den Meinungsstand bei Brosius-Gersdorf (Fn. 47), Art. 6 GG Rn. 57.

59 BVerfGE 48, 327 (339); ihm folgend G. Kirchhof, Der besondere Schutz der Familie in Art. 6 Abs. 1 des Grundgesetzes, AöR 2004, S. 542 (550 f.).

60 EGMR NJW 1979, 2449 (2452).

61 Wie hier Brosius-Gersdorf (Fn. 47), Art. 6 GG Rn. 112; Coester-Waltien (Fn. 47), Art. 6 GG Rn. 11 , 35; Robbers, in: von Mangoldt/Klein/Starck (Hrsg.), Grundgesetz, Art. 6 GG Rn. 86, 88. Siehe auch S. Sachs, Geschwister im Familienrecht, Tübingen 2007, S. 35 ff.

62 BVerfGE 8, 210 (215); BVerfGE 24, 119 (135); BVerfGE 25, 167 (196). 


\section{Die Rechtsstellung des nichtehelichen Vaters}

Der Schutz der nichtehelichen Eltern-Kind-Beziehung bezog sich in den 1950er und 1960er Jahren ausdrücklich nicht auf den Vater, was insofern konsequent war, als dieser nach damaligem Familienrecht als nicht mit dem Kind verwandt galt ( $\$ 1589$ Abs. 2 BGB i.d.F. 1900-1970). Erst mit der Reform des Nichtehelichenrechts im Jahr 1970 wurde die gesetzliche Fiktion der Nicht-Verwandtschaft abgeschafft. ${ }^{63}$ Seither wird nicht mehr bestritten, dass auch die Gemeinschaft aus nichtehelichen Kindern mit ihren beiden Eltern von Art. 6 Abs. 1 GG geschützt wird. ${ }^{64}$

Problematisch wurde dann aber die Verteilung des Sorgerechts zwischen den nichtehelichen Elternteilen. Das BGB-Familienrecht blieb über lange Zeit von der Vorstellung geprägt, nichteheliche Väter seien an der Pflege und Erziehung ihrer Kinder überwiegend nicht beteiligt. Dementsprechend sah das Nichtehelichengesetz für sie die Rolle des Zahlvaters vor: Das Kind erhielt Unterhalts- und Erbansprüche, der nichteheliche Vater erwarb aber keinerlei sorgerechtlichen Befugnisse. Er konnte lediglich Auskunft über die persönlichen Verhältnisse des Kindes verlangen und unter engen Voraussetzungen ein Umgangsrecht geltend machen. Erst im Jahr 1998 wurde mit der Familienrechtsreform die Möglichkeit eines gemeinsamen Sorgerechts der nichtehelichen Eltern eingeführt, das bis heute durch übereinstimmende Sorgeerklärungen gegenüber dem Standes- oder Jugendamt begründet werden kann $(\mathbb{1} 1626 \mathrm{a}$ BGB).

Mit dieser Regelung wurde allerdings der Mutter des nichtehelichen Kindes - anders als der Ehefrau - faktisch ein Vetorecht über das Sorgerecht des Vaters erteilt: War sie nicht gewillt, eine Erklärung zugunsten des gemeinsamen Sorgerechts abzugeben, konnte der nichteheliche Vater nicht sorgeberechtigt werden. Das Bundesverfassungsgericht bewertete dieses Bestimmungsrecht der Mutter im Jahr 2003 zunächst als verfassungsgemäß. Zwar werde der Mutter hier per Gesetz ein Alleinentscheidungsrecht verliehen. Aber:

„Der Gesetzgeber durfte davon ausgehen, dass eine Mutter, gerade wenn sie mit dem Vater und dem Kind zusammenlebt, sich nur ausnabmsweise und nur dann dem Wunsch des Vaters nach einer gemeinsamen Sorge verweigert, wenn sie dafür schwerwiegende Gründe hat, die von der Wabrung des Kindeswobls getragen werden, dass sie also die Möglichkeit der Verweigerung einer Sorgeerklärung nicht etwa als Machtposition gegenüber dem Vater missbraucht." 65

Wie das Bundesverfassungsgericht zu dieser Einschätzung kam, bleibt ungewiss, da es weder empirische Daten noch andere Argumente dafür anführt. Statt dessen zeigt

63 Gesetz über die rechtliche Stellung der nichtehelichen Kinder v. 19.8.1969, BGBl. I 1969, S. 1243, in Kraft getreten am 1.7.1970.

64 St. Rspr., d. BVerfG, vgl. BVerfGE 106, 166 (176); Schumann, Die nichteheliche Lebensgemeinschaft (Fn. 57), Rn. 235.

65 BVerfGE 107, 150 (177). 
die Entscheidung, wie tief die traditionellen geschlechtsbezogenen Vorstellungen von Elternschaft in dem verfassungsrechtlichen Bild der Familie bis in die heutige Zeit verankert sind. Die Mutter wird als der einzig sichere Elternteil des nichtehelichen Kindes dargestellt, und ihr wird eine beeindruckende Fremdnützigkeit im Handeln zugeschrieben. Der (nichteheliche) Vater hingegen erscheint als unsicherer Kantonist, dessen Wunsch nach sorgerechtlicher Verantwortung sorgsamer Prüfung bedarf.

Anders bewertete diese Rechtslage der Europäische Gerichtshof für Menschenrechte, der das de-facto-Vetorecht der Mutter im Jahr 2009 als Verstoß gegen die Rechte des nichtehelichen Vaters auf Achtung seines Privat- und Familienlebens (Art. 8 Abs. 1 EMRK) wertete. ${ }^{66}$ Der Gerichtshof äußerte erhebliche Zweifel an der Auffassung des Bundesverfassungsgerichts, die Mutter werde ihr Entscheidungsrecht nur zugunsten des Kindes verwenden. ${ }^{67}$ Diese Skepsis wird speziell für die deutsche Rechtslage durch eine breit angelegte rechtstatsächliche Untersuchung der nichtehelichen Elternschaft gestützt. ${ }^{68}$ Sie hat zum einen ergeben, dass ein hoher Anteil nichtehelicher Eltern sich die Sorgeverantwortung für ihre Kinder teilt. ${ }^{69}$ In diesen nichtehelichen Familien wiederum gibt es einen erheblichen Anteil an Vätern, die tatsächliche Sorgeverantwortung tragen, ohne ein Sorgerecht zu haben. Auf die Frage, warum sie keine Sorgeerklärung abgegeben haben, antwortete die Mehrheit von ihnen, sie hätten nicht gewusst, dass es so etwas gibt, sie hätten geglaubt, ein gesetzliches Sorgerecht zu haben, oder sie hätten es für einen unnötigen bürokratischen Schritt befunden, ihre Sorgeverantwortung rechtlich abzusichern. Die betroffenen Mütter hingegen, die keine Sorgeerklärung abgegeben hatten, begründeten dies zu einem nicht unerheblichen Teil damit, dass ihnen die Partnerschaft nicht stabil genug erschienen sei oder dass sie im Konfliktfall lieber allein entschieden. ${ }^{70}$ Die idealisierende Annahme, Mütter hätten für ihr Veto gegen das gemeinsame Sorgerecht stets schwerwiegende und kindeswohlrelevante Gründe, lässt sich auf dieser Datengrundlage schwerlich halten. So sieht es mittlerweile auch das Bundesverfassungsgericht, das unter anderem mit Bezug auf diese Befundlage seine Rechtsprechung änderte und das absolute Vetorecht der Mutter bei der Sorgeerklärung für verfassungswidrig erklärte. ${ }^{71}$ Inzwischen kann das Familiengericht auf Antrag des nichtehelichen Vaters

66 EGMR FamRZ 2010, 103 (Zaunegger./Deutschland).

67 Ebd., Ziff. 58.

68 K. Jurczyk/S. Walper. Vorgezogener Endbericht für das Projekt „Gemeinsames Sorgerecht nicht miteinander verheirateter Eltern“, 2010, http://www.bmj.de/SharedDocs/Downloads/DE/pdfs/Endbericht_Sorgerecht_final.pdf;jsessionid=D1189FEBE3C9DEEBC1ED8DE4075472FE.1_cid102? blob=publicationFile.

69 K. Jurczyk/S. Walper, Vorgezogener Endbericht (Fn. 68), S. 345: 62 \%, nach amtlichen Statistiken um $50 \%$.

70 K. Jurczyk/S. Walper, Vorgezogener Endbericht (Fn. 68), S. 146.

71 BVerfG FamRZ 2011, 452. 
über ein gemeinsames Sorgerecht entscheiden. ${ }^{72}$ Dennoch bleibt es bei einem gesetzlichen „Muttermodell“, in dem die Mutter das Sorgerecht qua Geburt erwirbt, während der nichteheliche Vater seinen Wunsch nach sorgerechtlicher Verantwortung ausdrücklich erklären muss. Wirklich gleichberechtigt wären Vater und Mutter in der nichtehelichen Elternbeziehung erst dann, wenn sie wie Eheleute gleich mit der Geburt des Kindes ein gemeinsames Sorgerecht erhielten. ${ }^{73}$

\section{Nichteheliche Elternschaft und medizinische Reproduktion}

Während die oben dargestellte Kontroverse um den Status des nichtehelichen Vaters überwiegend Konstellationen betrifft, in denen das Kind auf natürlichem Wege gezeugt wurde, bringen die medizinischen Reproduktionstechniken das verfassungsrechtliche Familienbild in noch anderer Weise ins Wanken. Oben wurde bereits darauf hingewiesen, dass in diesem Bereich nach wie vor ein vom Bundesverfassungsgericht bislang gebilligter Vorrang der Ehe gegenüber anderen Familienformen herrscht. Dieser Vorrang betrifft nicht nur die Kostenübernahme, sondern auch den Zugang zu medizinischen Reproduktionstechniken (a). Problematisch ist ferner die Elternschaft in diesen Konstellationen und damit der Schutz des Elternrechts aus Art. 6 Abs. 2 S. 1 GG (b).

\section{a. Der Zugang zu medizinischen Reproduktionstechniken}

Die künstliche Befruchtung ist im geltenden deutschen Recht bislang nur fragmentarisch geregelt: Das Embryonenschutzgesetz verbietet alle Verfahren, die zu einer gespaltenen Mutterschaft führen, also die Eizell- und Embryonenspende sowie die Leihmutterschaft ( $\mathbb{S} \mathbb{S}$ Abs. 1 Nr. 1, 6 und 7 ESchG). ${ }^{74}$ Die medizinische Reproduktion im Wege der Samenspende wird bislang durch das ärztliche Berufsrecht geregelt. Nach der einschlägigen Richtlinie der Bundesärztekammer steht die heterologe Samenspende - also die Befruchtung einer Frau mit dem Samen eines Mannes, mit dem

72 \$ 1626a BGB in der Fassung des Gesetzes zur Reform der elterlichen Sorge nicht miteinander verheirateter Eltern v. 16.4.2013, BGBl. I 2013, S. 795.

73 Vgl. zu unterschiedlichen Vorschlägen eines solchen „Elternmodells“ E. Schumann, Elterliche Sorge nicht miteinander verheirateter Eltern auf dem Prüfstand, FF 2010, S. 222-229; M. Coester, Sorgerechtliche Impulse aus Straßburg, NJW 2010, S. 482 (484); C. Holldorf, Sorgerecht nicht miteinander verheirateter Eltern, ZKJ 2010, S. 26 (27); M. Löhnig, Konsequenzen aus den Entscheidungen des Europäischen Gerichtshofs für Menschenrechte zum Sorgerecht des nicht mit der Kindesmutter verheirateten Vaters, FamRZ 2010, S. 338 (340 f.); S. Willutzki, Elterliche Sorge nicht miteinander verheirateter Eltern, ZKJ 2010, S. 86 (89) und die eingehende Untersuchung der unterschiedlichen Modelle bei D. Coester-Waltjen/V. Lipp/E. Schumann/B. Veit, Reformbedarf im nichtehelichen ElternKind-Verhältnis. 10. Göttinger Workshop zum Familienrecht 2011, Göttingen 2012.

74 Kritisch dazu J. Gernhuber/D. Coester-Waltjen, Familienrecht, 6. Aufl. München 2010, $\mathbb{5} 53$ Rn. 4-6. $\mathrm{Zu}$ Problemen der gespaltenen Mutterschaft in grenzüberschreitenden Fällen siehe VG Berlin, 15.4.2011, Az. VG 23 L 79/11; VG Berlin, 26.11.2009, Az. 11 L 396/09. Zur nach deutschem Recht grundsätzlich zulässigen Embryoadoption siehe E. Schumann, Familienrechtliche Fragen der Fortpflanzungsmedizin im Lichte des Grundgesetzes, in: H. Rosenau (Hrsg.), Für ein zeitgemäßes Fortpflanzungsmedizingesetz in Deutschland, Baden-Baden 2012, S. 155-201, insb. S. 166, 174, $196 \mathrm{f}$. 
sie nicht einer Partnerschaft lebt - grundsätzlich nur Eheleuten offen. ${ }^{75}$ Nicht verheiratete Frauen können nur behandelt werden, wenn sie in einer stabilen Beziehung mit einem nicht verheirateten Mann leben und dieser Mann die Vaterschaft des so gezeugten Kindes nach ärztlicher Einschätzung anerkennen wird. Alleinstehenden Frauen und Frauen, die in einer gleichgeschlechtlichen Lebensgemeinschaft leben, bleiben diese Methoden somit grundsätzlich verschlossen. Dieser Ausschluss ist aus gleichheitsrechtlicher Sicht fragwürdig. Nimmt man den Grundsatz der Gleichwertigkeit aller Familienformen ernst, so darf der Zugang zur medizinischen Reproduktion nicht von der formalen Verbindung der Betroffenen und auch nicht von ihrer konkreten Paarbeziehung abhängig gemacht werden, sondern es müsste sachliche Gründe für ihn geben. Das in diesem Zusammenhang häufig bemühte Kindeswohl kommt jedenfalls als pauschaler Rechtfertigungsgrund nicht in Frage, da nach bisheriger empirischer Erkenntnislage das Aufwachsen in einer gleichgeschlechtlichen Familie per se keine nachteiligen Wirkungen auf das Kind hat. ${ }^{76}$ Allenfalls im Einzelfall kann das staatliche Wächteramt aus Art. 6 Abs. 2 S. 2 GG eine begrenzte Vorwirkung entfalten, wenn sich in den ärztlichen Vorgesprächen abzeichnet, dass das erzeugte Kind unter Umständen aufwachsen müsste, die keinem Kind zugemutet werden können. ${ }^{77}$ Diese Einschränkung gilt jedoch für gleich- wie für gegengeschlechtliche Partnerschaften.

\section{b. Elternschaft nach künstlicher Befruchtung}

Ein weiteres Problem der heterologen Samenspende betrifft die Elternschaft: Ein auf diesem Wege gezeugtes Kind hat mehr als zwei Eltern: die leibliche Mutter und den Samenspender als leiblichen Vater sowie in heterosexuellen Konstellationen (zu gleichgeschlechtlichen Lebensgemeinschaften siehe unten E) den Mann, der die rechtliche Vaterschaft übernimmt, sei es qua Ehe ( $\$ 1592 \mathrm{Nr} .1$ BGB) oder durch Vaterschaftsanerkennung ( $\$ 1592$ Nr. 2 BGB). Problematisch ist die Dreierkonstellation sowohl für den Samenspender als auch für den rechtlich-sozialen Elternteil: Der Samenspender kann im Wege der Vaterschaftsfeststellung unterhaltspflichtig für das Kind werden, auch wenn er ursprünglich davon ausgegangen ist, dass mit der Spende keine familienrechtlichen Verpflichtungen einhergehen würden. Der rechtlich-soziale Elternteil kann aus seiner sorgenden Rolle gedrängt werden, wenn der Spender seinerseits die Vaterschaftsfeststellung verlangt. ${ }^{78}$

75 Bundesärztekammer, (Muster-)Richtlinie zur Durchführung der assistierten Reproduktion, Novelle 2006, Deutsches Ärzteblatt 103 (2006), S. A 1392-A 1403, hier Ziff. 3.1.1. Die Richtlinie ist in den meisten Bundesländern Bestandteil des landesrechtlichen ärztlichen Berufsrechts.

76 Vgl. M. Rupp (Hrsg.), Die Lebenssituation von Kindern in gleichgeschlechtlichen Lebensgemeinschaften, Köln 2009.; F. Wapler, Gleichgeschlechtliche Lebensgemeinschaften mit Kindern - verfassungsrechtliche Rahmenbedingungen, in: D. Funcke/P.Thorn (Hrsg.), Die gleichgeschlechtliche Familie mit Kindern. Interdisziplinäre Beiträge zu einer neuen Lebensform, Bielefeld 2010, S. 115 (138 ff.).

77 Vgl. Wapler (Fn. 76), S. $140 \mathrm{ff}$.

78 Für die Zulässigkeit eines solchen Anfechtungsverfahrens, auch wenn der Spender der Mutter nicht im natürlichen Sinn „beigewohnt“ hat, neuerdings BGH NJW 2013, 2589. 
Aus verfassungsrechtlicher Sicht ist in diesem Zusammenhang zu klären, in welchem Verhältnis leibliche und rechtliche Elternschaft zueinander stehen. Zunächst einmal deutet das Wort „natürlich“ in Art. 6 Abs. 2 S. 1 GG („Pflege und Erziehung der Kinder sind das natürliche Recht der Eltern [...]“) auf einen Vorrang der leiblichen vor der sozialen Elternschaft hin. Im einfachen Recht ist ein solcher jedoch nur bei der Mutter zu erkennen. Sie erhält die rechtliche Elternstellung ohne Weiteres mit der Geburt des Kindes ( $\$ 1591$ BGB). Die Elternschaft des Vaters hingegen ist in verschiedenen Familienkonstellationen unterschiedlich geregelt. Eine regelhafte Verbindung zwischen leiblicher Abstammung und rechtlicher Elternstellung besteht bei ihm gerade nicht.

Dennoch gibt das Wort „natürlich“ der leiblichen Elternschaft ein gewisses Gewicht: Der Verfassungsgeber hat damit deutlich gemacht, dass er an soziale Strukturen anknüpft, die der Staat nicht schafft, sondern lediglich schützt und fördert. Die traditionelle Vorstellung, dass Kinder auf natürliche Weise gezeugt und von ihren leiblichen Eltern gemeinsam aufgezogen werden, liegt dem Elternbegriff des Art. 6 Abs. 2 S. 1 GG folglich unausgesprochen zugrunde und ist bis heute nicht von einem Leitbild der rein sozialen Familie abgelöst worden. Wenn sich die Formen des Zusammenlebens auch massiv gewandelt haben, so ist leibliche Elternschaft immer noch ein wichtiger Faktor für die Zugehörigkeitsgefühle zwischen Kindern und Eltern. Der primäre Anknüpfungspunkt für eine rechtliche Regelung der Elternschaft ist daher aus verfassungsrechtlicher Sicht die leibliche Abstammung. ${ }^{79}$ Doch macht gerade die Betonung der mit der Elternschaft einhergehenden Verantwortung in Art. 6 Abs. 2 S. 1 GG deutlich, dass das Grundgesetz das Eltern-Kind-Verhältnis auch als ein gelebtes soziales Verhältnis im Blick hat. Die rechtliche und soziale Verantwortung für ein Kind kann ganz unabhängig von der leiblichen Verbindung bestehen, wenn der verantwortliche Erwachsene das Kind als sein eigenes akzeptiert und das Kind ihn als Elternteil erlebt. Die primäre Anknüpfung an die leibliche Abstammung kann daher durch Aspekte sozialer Bindung und Verantwortungsübernahme relativiert werden. ${ }^{80}$

Auch aus einer Abwägung der Grundrechte der Beteiligten ergibt sich kein eindeutiges Rangverhältnis zwischen leiblicher und sozialer Elternschaft. Kinder zu haben (oder nicht) ist ein wichtiger Aspekt der persönlichen Lebensgestaltung, der über Art. 2 Abs. 1 GG sowie über das Recht auf Familiengründung aus Art. 6 Abs. 1 GG verfassungsrechtlich geschützt wird. Die schützenswerte Bindung kann allerdings durch leibliche wie soziale Elternschaft begründet werden: Ein Mann, der jahrelang nicht wusste, dass eine Frau ein Kind von ihm bekommen hat, kann ein eminentes Interesse daran haben, dieses Kind zu kennen und eine Beziehung zu ihm aufzubauen. 
Andererseits aber kann ein sozialer Vater, der mit dem Kind zusammenlebt und die Verantwortung für sein Aufwachsen trägt, ein nicht minder schützenswertes Interesse daran haben, dass diese tatsächliche soziale Bindung und Verpflichtung im Recht als Eltern-Kind-Beziehung anerkannt und damit dauerhaft gesichert wird.

Aus der Perspektive des Kindes ist zunächst die soziale Bindung von entscheidender Bedeutung: Wer die soziale Verantwortung für sein Aufwachsen übernimmt, ist existenziell wichtig für das Kind. Das Interesse daran, gewachsene soziale Bindungen zu schützen, hat daher großes Gewicht für das Persönlichkeitsrecht des Kindes aus Art. 2 Abs. 1 i.V.m. Art. 1 Abs. 1 GG. Doch ist die leibliche Elternschaft für viele Kinder ebenfalls nicht bedeutungslos, denn sie ermöglicht ihm, die eigene Identität als Teil einer Abstammungskette und Familientradition zu begreifen. Nicht zuletzt aus diesem Grund ist das Recht auf Kenntnis der eigenen Abstammung als wichtiger Teil des allgemeinen Persönlichkeitsrechts des Kindes anerkannt. ${ }^{81}$ Aus verfassungsrechtlicher Sicht ist es folglich geboten, keine starre Rangfolge der Kriterien für die Bestimmung des Elternstatus und des verfassungsrechtlichen Elternrechts vorzusehen.

Für das einfache Recht bedeutet dies, dass der Gesetzgeber bei allen Regelungen, die Elternschaft betreffen, die Grundrechtspositionen aller Beteiligten sorgsam abwägen muss. Bedeutsam wird dies auch in Bezug auf die Rechtsstellung des Samenspenders. Hier stellt sich derzeit vor allem die Frage, ob eine Vereinbarung zwischen Samenspender und -empfänger dahingehend, dass aus der Samenspende keinerlei Rechte und Pflichten gegenüber dem Kind erwachsen, wirksam ist. In $\mathbb{\$} 1600$ Abs. 5 BGB ist für die Ehe bereits geregelt, dass der Spender in der Regel keinerlei Rechte und Pflichten gegenüber dem Kind geltend machen kann. Der Gesetzgeber ist, als er diese Vorschrift schuf, davon ausgegangen, dass der Samenspender mit seiner Mitwirkung an der assistierten Reproduktion regelmäßig konkludent auf seine rechtliche Vaterschaft und sein Anfechtungsrecht verzichtet. ${ }^{82}$ Diese Regelvermutung ist plausibel, da dem Samenspender in der Regel bekannt sein dürfte, dass seine Spende einem anderen Elternpaar den Kinderwunsch erfüllen soll. Verfassungsrechtlich scheint es nicht bedenklich, diesen Verzicht für alle Beteiligten rechtlich abzusichern. Wenn dies aber für Eheleute gilt, ist kaum einzusehen, wieso eine derartige Abrede nicht auch in anderen Familienkonstellationen gelten sollte. Strebt eine nichteheliche Lebensgemeinschaft eine heterologe Samenspende an, so ist die Interessenlage nicht grundsätzlich anders als in einer Ehe. Kommen die Beteiligten in dieser Konstellation überein, dass die Samenspende für den Spender ohne rechtliche Folgen bleiben soll, ist daher auch dies verfassungsrechtlich unbedenklich. ${ }^{83}$ Hiergegen ließe sich ein- 
wenden, dass man auf diese Weise dem Kind einen potentiell Unterhaltsverpflichteten raubt. Solange aber eine andere Person in diese Rolle eintritt, indem etwa der Partner der leiblichen Mutter die Vaterschaft anerkennt, hat das Kind zwei rechtliche Eltern, die in der umfassenden Verantwortung stehen. Mehr potentielle Elternteile als Kinder nach einer natürlichen Zeugung erwarten können, muss man auch außerhalb des Mutterleibes erzeugten Kindern nicht zur Verfügung stellen. Den Samenspender einer heterologen Insemination rechtlich von der Elternschaft auszuschließen - sofern er dem mit der Spende zugestimmt hat - trägt im Gegenteil der Realität heutiger Familienverhältnisse Rechnung, ohne den Grundrechtsschutz des Kindes zu verkürzen. ${ }^{84}$

\section{c. Multiple Elternschaft}

Die medizinische Reproduktion, aber nicht nur sie, wirft auch die Frage auf, wie viele rechtliche Eltern ein Kind haben kann. Das Problem stellte sich dem Bundesverfassungsgericht im Jahr 2003 anhand der folgenden Fallkonstellation: Ein Mann und eine verheiratete Frau hatten gemeinsam ein Kind gezeugt. Da das Kind in eine bestehende Ehe geboren wurde, wurde der Ehemann der Mutter automatisch zum rechtlichen Vater des Kindes ( $\$ 1592$ Nr. 1 BGB). Der leibliche Vater hatte in dieser Situation weder einen Anspruch auf Vaterschaftsanfechtung, noch auf ein Auskunfts- oder Umgangsrecht. Das Bundesverfassungsgericht billigte diese Rechtslage unter anderem mit dem Argument, ein Kind könne nur zwei Eltern im verfassungsrechtlichen Sinne, also als Träger des Elternrechts aus Art. 6 Abs. 2 S. 1 GG, haben. ${ }^{85}$ Das Elternrecht könne ferner nur Personen zustehen, die dem Kind rechtlich als Elternteil zugeordnet sind, weil nur dann gewährleistet ist, dass sie auch umfassend in der rechtlichen Elternpflicht stehen. ${ }^{86}$

In der familien- und verfassungsrechtlichen Literatur wird diese Zwei-Eltern-Theorie des Bundesverfassungsgerichts gelegentlich angezweifelt. ${ }^{87}$ Gegen sie wird angeführt, dass sie längst nicht mehr modernen Familienverhältnissen entspreche. In der Tat ist die Realität vieler Familien von gespaltener Elternschaft geprägt, nicht zuletzt in den vielfältigen Formen der Stief- oder Patchworkfamilie. Für das verfassungsrechtliche Zwei-Eltern-Modell spricht jedoch die enorme Bedeutung der Elternschaft für das Kind. Elternschaft ist eine Verantwortungsbeziehung, die zeitlich unbegrenzt besteht und grundsätzlich alle Belange des Kindes umfasst. Kinder brauchen Eltern, die sich voll für sie verantwortlich fühlen und denen sie verlässlich zugeordnet sind. Verant-

84 Wie hier J. Remus/D. Liebscher, Wohnst du noch oder sorgst du schon mit? - Das Recht des Samenspenders zur Anfechtung der Vaterschaft, NJW 2013, S. 2558 (2561); Schumann (Fn. 74), S. 197.

85 BVerfGE 108, 82 (101).

86 BVerfGE 108, 82 (101f.).

87 N. Dethloff, Regenbogenfamilien. Der Schutz von Eltern-Kind-Beziehungen in gleichgeschlechtlichen Partnerschaften, in: A. Söllner (Hrsg.), Gedächtnisschrift für Meinhard Heinze, München 2005, S. 133 (142); Brosius-Gersdorf (Fn. 47), Art. 6 GG Rn. 150. 
wortung aber droht entwertet zu werden, wenn sie auf zu viele Schultern verteilt wird. Könnte ein Kind drei oder gar vier Elternteile haben, die ihm gleichberechtigt rechtlich zugeordnet sind und den verfassungsrechtlichen Schutz des Art. 6 Abs. 2 S. 1 GG genießen, potenzierte sich das schon im klassischen Zwei-Eltern-Modell nicht unerhebliche Konfliktpotenzial. Man läse möglicherweise zu viel in die Verfassung hinein, wenn man das Zwei-Eltern-Modell als verfassungsimmanent beschreiben wollte, schützt Art. 6 Abs. 2 S. 1 GG doch primär die Beziehung jedes einzelnen Elternteils zum Kind und kein Elternkollektiv. ${ }^{88}$ Jedenfalls aber ist es verfassungsrechtlich gerechtfertigt, dem Abstammungsrecht das Zwei-Eltern-Modell zugrunde zu legen und eine Erweiterung auf mehr Personen auszuschließen.

Diese Argumentation bezieht sich nur auf die rechtliche Zuordnung der Elternstellung, nicht auf die Ausgestaltung des Sorgerechts und der Umgangsbeziehungen. Auf der einfachrechtlichen Ebene des Sorge- und Umgangsrechts sind Mehr-Ebenen-Modelle mit abgestufter Verantwortlichkeit durchaus möglich, etwa in der Form des „kleinen Sorgerechts“ in Alltagsangelegenheiten ( $\mathbb{S} 1687$ Abs. 1, 1687b Abs. 1 BGB, $\int 9$ Abs. 7 LPartG). ${ }^{89}$

\section{Der Wunsch nach einem Familienleben: die Rechtsstellung des leiblichen, aber nicht rechtlichen Vaters}

Erkennt man mit dem Bundesverfassungsgericht an, dass ein Kind nur zwei verfassungsrechtlich voll verantwortliche Eltern haben kann, so ist noch nicht entschieden, nach welchen Kriterien diese beiden Personen aus einer Dreier- oder gar Viererkonstellation ausgewählt werden. Oben wurde bereits erwähnt, dass bei einer solchen Entscheidung eine Vielzahl an Interessen zu berücksichtigen sind: die aller betroffenen möglichen Elternteile sowie des Kindes. Letztere müssen in der Abwägung besonderes Gewicht erhalten, da das Kind keinerlei Einfluss darauf hat, in welche Familienkonstellation es geboren wird. Umgekehrt proportional zu dieser fehlenden Handlungs- und Entscheidungsmacht steht das existenzielle Bedürfnis des Kindes nach stabilen und kontinuierlichen familiären Bezügen. Diese fundamentale Abhängigkeit jedenfalls des kleinen Kindes rechtfertigt, seine Belange in der Abwägung vorrangig zu berücksichtigen. Aus diesem Grund gibt das deutsche Familienrecht der sozial-familiären Beziehung des Kindes zu einem rechtlich-sozialen Elternteil grundsätzlich den Vorrang gegenüber den leiblichen Abstammungsverhältnissen. In anderen Worten: Die gelebte soziale Beziehung zu einem rechtlichen Elternteil, von dem

88 St. Rspr. d. BVerfG, vgl. BVerfGE 47, 46 (76); BVerfGE 99, 145 (164); BVerfG NJW 2013, 847, Ziff. 51.

89 Vgl. Dethloff (Fn. 87), S. 142 und dies, Kindschaftsrecht des 21. Jahrhunderts, ZJK 2009, S. 141 (144); R. Kemper, Rechtsanwendungsprobleme bei der eingetragenen Lebenspartnerschaft, FF 2001, S. 156 (161); M. Löhnig, Neue Partnerschaften der gemeinsam sorgeberechtigt gebliebenen Eltern - Welche Rechte haben die neuen Partner?, FPR 2008, S. 157 (159); K. Muscheler, Das Recht der Stieffamilie, FamRZ 2004, S. 915 (920 f.). Siehe auch den Überblick bei Wapler (Fn. 76), S. 150 ff. 
das Kind nicht abstammt, ist in aller Regel schützenswerter als eine leibliche Abstammungsbeziehung, die von keiner sozialen Bindung begleitet wird. ${ }^{90}$ Auch dieser Grundsatz ist allerdings nicht abwägungsfest. In den letzten Jahren haben insbesondere die Rechte des leiblichen, aber nicht rechtlichen Vaters in Rechtsprechung und Gesetzgebung stärkeres Gewicht erhalten. Der Grund dafür war wie auch schon beim Sorgerecht eine eklatante Ungleichbehandlung leiblicher Mütter und Väter im Familienrecht des BGB: Während die leibliche Mutter eines Kindes seit jeher gem. \$1591 BGB mit der Geburt auch rechtliche Mutter wird, muss der leibliche Vater in einer nichtehelichen Beziehung seine Vaterschaft anerkennen bzw. feststellen lassen. Ist bereits ein anderer Mann der rechtliche Vater des Kindes, etwa als Ehemann der Mutter gem. $\$ 1592$ Nr. 1 BGB, kann diese Vaterschaft nur durch Anfechtung wieder beseitigt werden. Der leibliche Vater aber hatte zunächst kein solches Anfechtungsrecht, ihm war der Zugang zur rechtlichen Vaterschaft in diesen Fällen mithin kategorisch versperrt. Das Bundesverfassungsgericht stellte im Jahr 2003 fest, dass damit gegen Grundrechte des nichtehelichen Vaters verstoßen wurde: Zwar sei dieser nicht Träger des Elternrechts aus Art. 6 Abs. 2 S. 1 GG, da er dem Kind nicht rechtlich als Elternteil zugeordnet sei. Wohl aber werde mit dieser Grundrechtsnorm auch sein Interesse daran, rechtlicher Vater seines leiblichen Kindes zu werden, geschützt. Das Recht müsse ihm daher ein Verfahren zur Verfügung stellen, mit dem er seine Vaterschaft geltend machen kann. ${ }^{91}$ In ähnlicher Weise sieht auch der Europäische Gerichtshof für Menschenrechte den Wunsch nach einem Familienleben mit dem leiblichen Kind (intended family life) als Teil des konventionsrechtlich geschützten Rechts auf Privat- und Familienleben an. ${ }^{92}$

Der deutsche Gesetzgeber hat auf diese Rechtsprechung mit zwei Änderungen reagiert: Zum einen ist der leibliche, aber nicht rechtliche Vater seit 2004 berechtigt, die Vaterschaft des rechtlichen Vaters anzufechten, allerdings nur dann, wenn zwischen diesem und dem Kind keine sozial-familiäre Beziehung besteht $(\mathbb{\$} 1600 \mathrm{Abs.} 2$ BGB). ${ }^{93}$ Der Schutz der sozialen Bindungen des Kindes geht hier also konsequent vor, was der grundrechtlichen Abwägungslage entspricht und daher verfassungs- und auch konventionsrechtlich nicht zu beanstanden ist.

Nach dem 2013 eingeführten $\$$ 1686a BGB kann ein Vater darüber hinaus unter bestimmten Umständen Auskunft verlangen und ein Umgangsrecht erhalten. ${ }^{94}$ Die gesetzliche Regelung bleibt gegenüber der Rechtsprechung des Bundesverfassungs-

90 BVerfGE 108, 82 (106), BVerfG NJW 2013, 847, Ziff. 59.

91 BVerfGE 108, 82.

92 Siehe EGMR, NJW 2011, 3565 (Anayo/Deutschland), Ziff. 71; EGMR FamRZ 2011, 1715 (Schneider/Deutschland), Ziff. 99; EGMR, 22.3.2012, Nr. 23338/09 (K./Deutschland), Ziff. 77.

93 Gesetz zur Änderung der Vorschriften über die Anfechtung der Vaterschaft und das Umgangsrecht von Bezugspersonen des Kindes v. 23.4.2004, BGBl. I 2004, S. 598, in Kraft getreten am 30.4.2004.

94 Gesetz zur Stärkung der Rechte des leiblichen, aber nicht rechtlichen Vaters v. 4.7.2013, BGBl. I 2013, S. 2176. 
gerichts insofern restriktiv, als der Anspruch überhaupt nur besteht, wenn der Vater zuvor ein „nachhaltiges Interesse am Kind“ bezeugt hat. Nach der Rechtsprechung des Bundesverfassungsgerichts reicht es für den eingeschränkten Schutz des intendierten Familienlebens hingegen aus, dass eine genetische Verwandtschaft zwischen Vater und Kind besteht. ${ }^{95}$ Aus verfassungsrechtlicher Sicht muss der leibliche Vater also kein nachhaltiges Interesse an seinem Kind haben, um jedenfalls einen Minimalschutz seiner Elternposition zu erlangen. Auch nach konventionsrechtlichen Grundsätzen ist der unwissende, verunsicherte oder im Vorfeld desinteressierte leibliche Vater über die Achtung des Privatlebens aus Art. 8 Abs. 1 EMRK mit seinem gegenwärtigen Wunsch nach einem Familienleben mit seinem Kind geschützt. ${ }^{96} \mathrm{Al}-$ lerdings dürfte ein Umgangsanspruch eines desinteressierten Vaters aus anderen Gründen ausgeschlossen sein: Aus der Perspektive des Kindes ist der Umgang mit einem Menschen, der sich für seine Person nicht interessiert, sondern den Umgang aus anderen Gründen geltend macht - etwa, um die Mutter zurückzugewinnen, Macht über sie auszuüben oder um sich an der Mutter und ihrem Ehemann für erlittene Kränkungen zu rächen - in aller Regel nicht förderlich und kann folglich auch dem Kindeswohl nicht dienen. Auch ohne das Kriterium des „nachhaltigen Interesses" könnte das Kind folglich vor Umgangskontakten geschützt werden, die sein Wohl nicht befördern.

\section{E. Gleichgeschlechtliche Familien}

Eine erhebliche Herausforderung für das verfassungsrechtliche Bild der Familie sind gleichgeschlechtliche Lebensgemeinschaften mit und ohne Kinder. Noch in der erwähnten Entscheidung aus dem Jahr 2003, in der das Bundesverfassungsgericht seine Zwei-Eltern-Theorie explizierte, stellte es lapidar fest, Träger des Elternrechts könnten nur „eine Mutter und ein Vater“97 sein. Bei näheren Hinsehen wollte es mit dieser Aussage nicht explizit der gleichgeschlechtlichen Elternschaft eine Absage erteilen, ging es doch lediglich darum, die Zahl potentieller Träger des Elternrechts auf zwei zu begrenzen. Für das Bundesverfassungsgericht lag es seinerzeit auf der Hand, dass diese beiden Elternteile notwendig verschiedengeschlechtliche Personen sein mussten, wie es der natürlichen Zeugungssituation entspricht und im gesamten damaligen Familienrecht als selbstverständlich vorausgesetzt wurde. ${ }^{98}$ Nicht einmal zwei Jahre

95 BVerfGE 108, 82 (101): „Der Schutz des Art. 6 Abs. 2 Satz 2 GG setzt die rechtliche Elternschaft nicht voraus. Der Mann, von dem ein Kind abstammt, ist Vater des Kindes, auch wenn er von der Rechtsordnung nicht als solcher anerkannt ist. Mehr als diese auf Abstammung beruhende Elternschaft setzt Art. 6 Abs. 2 Satz 1 GG für die Einbeziehung von Eltern in seinen Schutzbereich nicht voraus. Allerdings macht dies allein noch nicht den biologischen Vater neben dem rechtlichen Vater zum Träger des Elternrechts.".

96 EGMR, NJW 2011, 3565 (Anayo/Deutschland), Ziff. 71; EGMR FamRZ 2011, 1715 (Schneider/ Deutschland), Ziff.99; EGMR, 22.3.2012, Nr. 23338/09 (K./Deutschland), Ziff. 77.

97 BVerfGE 108, 82 (101).

98 Vgl. die entsprechenden Ausführungen in BVerfG NJW 2013, 847, Ziff. 52. 
später wurde gleichgeschlechtlichen Paaren im Lebenspartnerschaftsgesetz die Stiefkindadoption ermöglicht ( $\$ 9$ Abs. 7 LPartG) $){ }^{99}$ Ein Lebenspartner kann seither das Kind des anderen Lebenspartners annehmen - mit der Konsequenz, dass das Kind anschließend personenstandsrechtlich zwei Väter oder zwei Mütter hat. Der Gesetzgeber hat mit dieser Änderung die gleichgeschlechtliche Lebensgemeinschaft mit Kindern als rechtlich vollwertige Familie anerkannt - das Bundesverfassungsgericht hat sich dieser Bewertung im Jahr 2013 explizit angeschlossen, als es die Ungleichbehandlung der Lebenspartnerschaft bei der Sukzessivadoption rügte. ${ }^{100}$

Sukzessivadoption bedeutet, dass zunächst ein Partner ein Kind allein annimmt, und anschließend der andere Partner dieses Kind ebenfalls adoptiert. Für Eheleute ist diese Möglichkeit in $\$ 1742$ BGB eröffnet, nicht aber für Lebenspartner. Das Bundesverfassungsgericht erkannte darin eine ungerechtfertigte Diskriminierung der Lebenspartnerschaft gegenüber der Ehe. Der Gesetzgeber muss nun bis Juni 2014 eine verfassungskonforme Regelung schaffen; bis dahin ist die Sukzessivadoption in eingetragenen Lebenspartnerschaften zu akzeptieren. Das Bundesverfassungsgericht öffnet sich damit der empirischen Erkenntnis, nach der gleichgeschlechtliche Eltern für das Kind nicht grundsätzlich nachteiliger sind als gegengeschlechtliche. Vor diesem Hintergrund kann auch das nach wie vor bestehende Verbot der gemeinschaftlichen Adoption durch Lebenspartner nur als gleichheitswidrig bewertet werden. ${ }^{101}$

Bis es soweit kommen konnte, dass in der Rechtswissenschaft ernsthaft über die Adoption von Kindern in gleichgeschlechtlichen Lebensgemeinschaften diskutiert werden konnte, war ein weiter Weg zu gehen. Schon die Einführung der Lebenspartnerschaft als rechtlich formalisierte gleichgeschlechtliche Lebensgemeinschaft war von heftigen verfassungsrechtlichen Debatten begleitet. In einer ersten Phase betraf sie den Begriff der Ehe. Im Grundgesetz wird die Ehe nicht definiert, so dass es durchaus denkbar wäre, auch homosexuelle Lebensgemeinschaften unter den Ehebegriff zu fassen. Das zentrale Merkmal der Ehe wäre dann die auf Dauer angelegte und rechtlich formalisierte Verantwortungs- und Einstehensgemeinschaft zweier Menschen, nicht aber die Verschiedengeschlechtlichkeit. Das Bundesverfassungsgericht hat sich dieser Auffassung nicht angeschlossen, sondern in einem Kammerbeschluss aus dem Jahr 1993 die Verschiedengeschlechtlichkeit der Partner als begriffsbestimmendes Merkmal der Ehe bezeichnet. Sein zentrales Argument ist die Wortgebrauchsge-

99 Gesetz zur Überarbeitung des Lebenspartnerschaftsrechts v. 15.12.2004, BGBl. I 2004, S. 3396, in Kraft seit 1.1.2005.

100 BVerfG NJW 2013, 847, Ziff. 60: „Die sozial-familiäre Gemeinschaft aus eingetragenen Lebenspartnern und dem angenommenen Kind eines Lebenspartners bildet eine durch Art. 6 Abs. 1 GG geschützte Familie [...]." Siehe in diesem Sinne in Bezug auf Art. 8 EMRK auch schon EGMR, 24.7.2003, Rs. 40016/98 (Karner/Österreich).

101 Wie hier Brosius-Gersdorf (Fn. 47), Art. 6 GG Rn. 84, 120; siehe dazu ausführlich Wapler (Fn. 76), S. 134 ff. In dem am 14.3.2014 vorgestellten Regierungsentwurf „zur Umsetzung der Entscheidung des Bundesverfassungsgerichts zur Sukzessivadoption durch Lebenspartner“ ist die Volladoption hingegen nicht vorgesehen. 
schichte, nach der das Wort „Ehe“ im familienrechtlichen Kontext in Deutschland stets im Sinne einer Lebensgemeinschaft von Mann und Frau verstanden wurde. Ein grundlegender Wandel dieses Begriffsverständnisses sei für die Zukunft nicht ausgeschlossen, in der Gegenwart jedoch nicht zu erkennen. ${ }^{102}$

Nachdem diese Frage zumindest vorläufig als geklärt gelten musste, ${ }^{103}$ diskutierte man, ob die dauerhafte Lebensgemeinschaft gleichgeschlechtlicher Paare als eigenes Rechtsinstitut neben der Ehe formalisiert werden durfte. Der Gesetzgeber entschloss sich im Jahr 2001 zu diesem Schritt und schuf die Lebenspartnerschaft als ein solches eheähnliches Institut. ${ }^{104}$ Die Verfassungsmäßigkeit des Lebenspartnerschaftsgesetzes wurde in der Folge mit dem Argument angezweifelt, das verfassungsrechtlich notwendige „Abstandsgebot“ zur Ehe sei verletzt. Dem liegt die Annahme zugrunde, dass der verfassungsrechtliche Schutz der Ehe nur dann möglich ist, wenn die Ehe gegenüber allen anderen Lebensgemeinschaften rechtlich privilegiert bleibt. ${ }^{105}$ Die beiden Senate des Bundesverfassungsgerichts waren sich in dieser Frage zunächst nicht einig: Während der erste Senat ein eherechtliches Abstandsgebot ausdrücklich verneinte, ${ }^{106}$ ging der zweite Senat von einem „Differenzierungsgebot“ zugunsten der Ehe aus. ${ }^{107}$ Dem widersprach der erste Senat in einer weiteren Entscheidung und hielt an seiner Auffassung fest. ${ }^{108}$ In der Tat ist wenig einsichtig, dass der Schutz einer sozialen Gemeinschaft zwingend die Schlechterstellung anderer Lebensweisen erfordert. Vielmehr sollten sich Schutz und Förderung daran orientieren, was die Ehe benötigt, um ihren Zweck - die Absicherung einer dauerhaften Verantwortungsgemeinschaft - zu erfüllen. Gleiches muss dann für andere soziale Gemeinschaften gelten, die rechtlich formalisiert sind und damit ebenfalls unter dem Schutz des Rechts stehen. Die Regelungen der Ehe und der eingetragenen Lebenspartnerschaft sind daher an allgemeinen gleichheitsrechtlichen Maßstäben zu messen, das heißt, eine Un-

102 BVerfG NJW 1993, 3058.

103 Bis heute die überwiegende Auffassung im Schrifttum, vgl. den Überblick über den Meinungsstand bei Coester-Waltien (Fn. 47), Art. 6 GG Rn. 9. Siehe aber S. Rixen, Das Ende der Ehe? Neukonturierung der Bereichsdogmatik von Art. 6 Abs. 1 GG: ein Signal des spanischen Verfassungsgerichts, JZ 2013, S. 864-873.

104 Gesetz über die eingetragene Lebenspartnerschaft (Lebenspartnerschaftsgesetz) vom 16.2.2001, BGBl. 2001 I, S. 266.

105 Für ein „Abstandsgebot“ z.B. W. Pauly, Sperrwirkungen des verfassungsrechtlichen Ehebegriffs, NJW 1997, S. 1055; R. Scholz/A. Uhle, „Eingetragene Lebenspartnerschaft“ und Grundgesetz, NJW 2001, S. 393; G. Krings, Die „eingetragene Lebenspartnerschaft“ für gleichgeschlechtliche Paare, ZRP 2000, S. 409-414; M. Burgi, Schützt das Grundgesetz die Ehe vor der Konkurrenz anderer Lebensgemeinschaften?, Der Staat 2000, S. 487; aus dem neueren Schrifttum siehe K. Pfizenmayer, Die Rechtsstellung gleichgeschlechtlicher Lebenspartner in der deutschen Rechtsordnung, Hamburg 2007, S. $86 \mathrm{ff}$.

106 BVerfGE 105, 313 (348).

107 BVerfG DVBl. 2007, 1432.

108 Vgl. BVerfG DVBl. 2009, 1513: „[...] aus der Befugnis, in Erfüllung und Ausgestaltung des verfassungsrechtlichen Förderauftrags die Ehe gegenüber anderen Lebensformen zu privilegieren, lässt sich kein in Art. 6 Abs. 1 GG enthaltenes Gebot herleiten, andere Lebensformen gegenüber der Ehe zu benachteiligen." Im Ergebnis ebenso Brosius-Gersdorf (Fn. 47), Art. 6 GG Rn. 82. 
gleichbehandlung der einen Gemeinschaft gegenüber der anderen bedarf eines rechtfertigenden Grundes. ${ }^{109}$ Legt man diesen Maßstab an, so zeigt sich, dass die grundrechtliche Interessenlage in beiden Instituten vollkommen identisch ist: In beiden Formen der Zweierbeziehung geht es darum, die gegenseitige Verantwortlichkeit der Partner rechtlich abzusichern, etwa durch Unterhalts- und Erbansprüche und durch Entscheidungsbefugnisse im Not- und Krankheitsfall. Auch das häufig bemühte Argument, nur die Ehe sei darauf angelegt, Kinder zu bekommen und aufzuziehen, kann nicht überzeugen, da de facto in Ehen wie in Lebenspartnerschaften Kinder aufwachsen und vergleichbare Interessen an rechtlicher Absicherung der Sorgeverantwortung bestehen. Angesichts dieser strukturellen Ähnlichkeiten lässt sich durchaus fragen, ob es nicht sinnvoller wäre, die dauerhafte und auf gegenseitige Verantwortung angelegte Zweierbeziehung unter Erwachsenen in einem einzigen Rechtsinstitut zu erfassen, wie es etwa im spanischen und im niederländischen Recht geregelt ist. ${ }^{110}$ Die Rechtswirklichkeit in Deutschland entwickelt sich allerdings derzeit eher dahin, dass es bei zwei Rechtsinstituten - Ehe und Lebenspartnerschaft - bleibt, die sich in ihrem Gewährleistungsgehalt zunehmend decken.

\section{F. Das Kind als Familienmitglied}

Wie Familie in der Wirklichkeit gelebt wird, hängt in erheblichem Maße davon ab, wie die erwachsenen Familienmitglieder ihr Zusammenleben gestalten. Erheblich gewandelt hat sich im Laufe der letzten Jahrzehnte aber auch der Status des Kindes in der Familie, der im Folgenden nur insoweit thematisiert wird, als sich aus ihm Rückschlüsse auf den verfassungsrechtlichen Familienbegriff ergeben.

\section{Das Recht des Kindes auf Entwicklung zu einer selbstbestimmten Person}

Dass auch das Kind Träger der Grundrechte ist, ergibt sich schon aus dem Wortlaut dieser Rechte, die - mit Ausnahme des Wahlrechts (Art. 38 Abs. 1 GG) - keine Altersbeschränkung enthalten. Unmittelbar nach Inkrafttreten des Grundgesetzes herrschte die Auffassung vor, Kinder würden auch im Gebrauch ihrer Grundrechte umfassend von den Eltern vertreten. ${ }^{111}$ Dies gipfelte in der aus heutiger Sicht absurden Vorstellung, eine Heimunterbringung gegen den Willen des Kindes, aber mit dem Einverständnis seiner Eltern sei als „freiwillig“ zu qualifizieren. ${ }^{112}$ Die rechtliche

109 Vgl. nur die beiden jüngsten Entscheidungen des BVerfG zur Sukzessivadoption (BVerfG NJW 2013, 847) und zum Ehegattensplitting (7.5.2013, 2 BvR 1981/06); wie hier Brosius-Gersdorf (Fn. 47), Art. 6 GG Rn. 83; S. Stüber, Vom Gebot, die Ehe zu fördern, FPR 2006, 117 (119).

110 Vgl. Art. 44 Código Civil (Spanien); Art. 1:30 Burgelijk Wetboek (Niederlande). Zur Möglichkeit nach deutschem Recht, die Ehe für gleichgeschlechtliche Lebensgemeinschaften zu öffnen siehe $R i$ xen (Fn. 103); V. Beck, Gleichstellung durch Öffnung der Ehe, FPR 2013, S. 220-226; Brosius-Gersdorf (Fn. 47), Art. 6 GG Rn. 81.

111 H. Peters, Elternrecht, Erziehung, Bildung und Schule, in: K.A. Bettermann/H.C. Nipperdey (Hrsg.), Die Grundrechte, Bd. 4.1, Berlin 1960, S. 395.

112 W. Maurer, Das private Unterbringungsrecht und Art. 104 GG, FamRZ 1960, S. 468 (472). 
Vertretung des Kindes wurde demnach als Vertretung im Willen angesehen, mit dem Ergebnis, dass der Wille der Eltern den des Kindes ersetzte. Von dieser Haltung rückte das Bundesverfassungsgericht etwa ab den späten 1960er Jahren ab. Da das Kind Grundrechtsträger mit eigener Menschenwürde und einem eigenen Recht auf Entfaltung seiner Persönlichkeit ist, ${ }^{113}$ hat es nicht nur ein Recht auf (paternalistischen) Schutz, sondern auch auf alters- und reifeabhängige Beteiligung an eigenen Angelegenheiten. ${ }^{114}$ In der Struktur der heutigen Grundrechtsdogmatik fließt dieses Recht aus dem allgemeinen Persönlichkeitsrecht des Kindes (Art. 2 Abs. 1 i.V.m. Art. 1 Abs. 1 GG); es wird zudem seit 1992 durch Art. 12 Abs. 1 der UN-Kinderrechtskonvention verstärkt. ${ }^{115}$ Das allgemeine Persönlichkeitsrecht des Kindes ist folglich als ein Recht des Kindes auf Entwicklung zu einer selbstbestimmten Persönlichkeit zu verstehen, das ihm wachsende Mit- und Selbstbestimmungsrechte verleiht. ${ }^{116}$ Das Kind tritt dadurch als Subjekt mit einer eigenen Perspektive aus dem Familienverband heraus und gerät als Individuum in den Blick des Verfassungsrechts.

\section{Das Recht des Kindes auf Schutz}

Die Beteiligungsrechte des Kindes kommen dessen Streben nach Autonomie und Entwicklung entgegen. Auf der anderen Seite stehen Abhängigkeit und Schutzbedürftigkeit des Kindes, die vor allem in den ersten Lebensjahren nicht weggeleugnet werden können. Der Schutz des Kindes ist nach Art. 6 Abs. 2 S. 1 GG primär den Eltern anvertraut. Erst wenn die Eltern ihn nicht mehr gewährleisten können, greift das staatliche Wächteramt des Art. 6 Abs. 2 S. 2 GG. Auch hier zeigt sich in der Verfassung das Bestreben, die Familie als Ort privatautonomer Selbstentfaltung bestmöglich vor staatlicher Einflussnahme zu schützen. Allerdings definiert das Grundgesetz nicht die Eingriffsschwelle für das staatliche Wächteramt. Im einfachen Recht wird sie mit dem Begriff der Kindeswohlgefährdung umschrieben (vgl. $\$ 1666$ Abs. 1 BGB), eine Terminologie, die sich auch in der Rechtsprechung des Bundesverfassungsgerichts und im verfassungsrechtlichen Schrifttum wiederfindet. ${ }^{117}$ Dabei sollte jedoch nicht übersehen werden, dass es aus verfassungsrechtlicher Sicht auch

113 St. Rspr. seit BVerfGE 24, 119 (144).

114 St. Rspr., vgl. BVerfGE 57, 171, Ziff. 29; BVerfG ZKJ 2008, 421, Ziff. 30; BVerfG, 18.5.2009, Az. 1 BvL 142/09; BVerfGE 121, 69.

115 Art. 12 Abs. 1 UN-KRK: „Die Vertragsstaaten sichern dem Kind, das fähig ist, sich eine eigene Meinung zu bilden, das Recht zu, diese Meinung in allen das Kind berührenden Angelegenheiten zu äußern, und berücksichtigen die Meinung des Kindes angemessen und entsprechend seinem Alter und seiner Reife.".

116 Ausführlich zur verfassungsrechtlichen Herleitung dieses Grundrechts F. Wapler, Kinderrechte und Kindeswohl. Eine Untersuchung zum Status des Kindes im öffentlichen Recht, Tübingen 2014 (im Erscheinen).

117 BVerfGE 60, 79, Rn. 43, st. Rspr.Aus dem Schrifttum Coester-Waltien (Fn. 47), Art. 6 GG Rn. 81; D. Umbach, in: ders./Thomas Clemens (Hrsg.), Grundgesetz. Mitarbeiterkommentar und Handbuch, Heidelberg 2002, Art. 6 GG Rn. 78; Badura (Fn. 53), Art. 6 GG Rn. 110, 139; Robbers (Fn. 61), Art. 6 GG Rn. 243; ausf. M. Jestaedt, in: Bonner Kommentar zum Grundgesetz, Heidelberg 1995, Art. 6 Abs. 2 GG Rn. 46. 
im Hinblick auf die schützenswerten Kindesbelange um eine Abwägung von Grundrechtspositionen geht: Das staatliche Wächteramt begründet eine Schutzpflicht des Staates zugunsten unverzichtbarer Rechtspositionen des Kindes, etwa seiner körperlichen Unversehrtheit. Diese Schutzpflicht realisiert sich immer nur dann, wenn die Eltern des Kindes den Schutz nicht selbst gewährleisten können. Das staatliche Wächteramt ist aus der Sicht des Verfassungsrechts folglich Grundrechtsschutz.

Diese Begrenzung des staatlichen Wächteramts auf Gefährdungssituationen wurde in den letzten Jahren zunehmend in Frage gestellt, und es wurde gefordert, staatliche Kontroll- und Eingriffsbefugnisse in die Familie zu erweitern. ${ }^{118}$ Diese Debatte berührt nicht nur den Status des Kindes, sondern auch den der Familie in der politischen Gemeinschaft fundamental. Hinter dem Ruf nach mehr staatlicher Einmischung stehen erhebliche Zweifel, ob die Familie ihre verfassungsrechtliche Aufgabe, Kindern den primären Sozialisations- und Bildungsort zu bieten, heute noch erfüllen kann. ${ }^{119}$ Der Parlamentarische Rat stattete Eltern mit einem großzügigen Vertrauensvorschuss aus, den das Bundesverfassungsgericht in ständiger Rechtsprechung mit der Formulierung umschreibt, Eltern seien regelmäßig die Personen, denen das Wohl ihrer Kinder am meisten am Herzen liege. ${ }^{120}$ Empirische Untersuchungen des modernen Familienlebens ergeben im Großen und Ganzen nichts anderes; im Gegenteil hat die Gewalt in den Familien abgenommen und verstehen sich die Generationen besser denn je. ${ }^{121}$ Das Bundesverfassungsgericht hält denn auch mit dem überwiegenden Teil des verfassungsrechtlichen Schrifttums daran fest, dass Eingriffe in das Elternrecht nur zulässig sind, wenn dem Kind eine konkrete Gefährdung droht. In mehreren Entscheidungen der letzten Jahre musste es Jugendämter und Gerichte daran erinnern, dass Kinder nicht schon aus ihren Familien genommen werden dürfen, wenn ein Gutachten aus dem Verhalten der Eltern auf mögliche zukünftige Entwicklungsschwierigkeiten schließt, ohne dass die Kinder aktuell Auffälligkeiten zeigen. ${ }^{122}$ Vor diesem Hintergrund gibt die dramatisch gestiegene Zahl der Inobhut-

118 Vgl. aus der verfassungsrechtlichen Literatur P. Hölbling, Wie viel Staat vertragen Eltern? Systematische Entfaltung eines gestuften Maßnahmenkonzeptes vor dem Hintergrund des Elterngrundrechts. Berlin 2010.

119 Vgl. Hölbling (Fn. 118), S. 31 ff.; W. Röchling, Neue Aspekte zu Kinderschutz und Kindeswohl? FamRZ 2007, S. 1775 (1779); M. Siegfried, Kindstötung: Der hohe Wächter- und Schutzauftrag des Jugendamts, FPR 2008, S. 264. Siehe zu dieser Problematik kritisch E. Schumann, Stärkung und Schutz des Kindeswohls, in: dies./V. Lipp/B. Veit (Hrsg.), Reform des familiengerichtlichen Verfahrens, Göttingen 2009, S. 229-258.

120 BVerfGE 59, 360 (376); aus der neueren Rechtsprechung BVerfGE 104, 373, Ziff. 41; BVerfGE 107, 150, Ziff. 62; BVerfG ZKJ 2011, 428, Ziff. 14; BVerfG ZKJ 2012, 186, Ziff. 21.

121 K. Bussmann, Das Recht auf gewaltfreie Erziehung aus juristischer und empirischer Sicht, FPR 2002, S. 289 (292 f.); K. Hurrelmann/S. Andresen, Kinder in Deutschland (World-Vision-Kinderstudie), Bonn 2007, S. 92 ff. (Verhältnis Eltern/Kinder), S. 102 ff. (Strafen). Siehe auch Schumann (Fn. 119), S. $240 \mathrm{ff}$.

122 BVerfG ZKJ 2011, 428; BVerfG ZKJ 2012, 186. 
nahmen ${ }^{123}$ ebenso Anlass zur Sorge wie die Tendenz, flächendeckende Überwachungen des Elternverhaltens einzuführen, etwa durch Kontrollen nach versäumten Vorsorgeuntersuchungen. Eine begleitende staatliche Erziehungskontrolle ohne äußeren Anlass ist verfassungsrechtlich nicht zulässig, sondern verletzt den Primat des Elternrechts. ${ }^{124}$

\section{Der Schutz der familiären Bindungen des Kindes}

Eltern sind nach Art. 6 Abs. 2 Satz 1 GG zur Pflege und Erziehung ihrer Kinder verpflichtet. Kontrovers diskutiert wurde vor einigen Jahren, ob dem ein verfassungsmäßiges Recht des Kindes auf Pflege und Erziehung korrespondiert. Ausgelöst wurde diese Diskussion durch eine Entscheidung des Bundesverfassungsgerichts, in der ein Recht des Kindes auf Pflege und Erziehung gegen seine Eltern postuliert wurde. ${ }^{125}$ Damit sprengte das Gericht ohne nähere Begründung den üblichen grundrechtsdogmatischen Rahmen: Art. 1 Abs. 3 GG verpflichtet alle staatliche Gewalt, aber eben auch nur staatliche Gewalt, die Grundrechte zu achten. Privatpersonen sind grundsätzlich nicht grundrechtsverpflichtet. In Teilen der staatsrechtlichen Literatur wird das Recht des Kindes auf Pflege und Erziehung gegen seine Eltern dennoch bis heute zitiert, auch wenn sich bislang niemand gefunden hat, der es mit stichhaltigen Argumenten untermauert hätte. ${ }^{126}$ Das Bundesverfassungsgericht selbst hat die Formulierung nicht wieder aufgegriffen. Statt dessen hat es in seiner Entscheidung zur Sukzessivadoption in gleichgeschlechtlichen Lebenspartnerschaften eine andere Konstruktion entwickelt: Das Recht des Kindes auf staatliche Gewährleistung der elterlichen Pflege und Erziehung. ${ }^{127}$ Der Staat hat demnach eine Schutzpflicht dahingehend, dass er rechtliche Bedingungen schaffen muss, die eine private Kindererziehung

123 Vgl. Th. Rauschenbach/J. Pothmann, Frühe Hilfen als aktiver Kinderschutz. Rückgang der Kindstötungen - Zunahme der Hilfen, KomDat 2/2010, S. 1 (2); der Trend setzt sich fort, siehe die aktuellen Zahlen des statistischen Bundesamtes unter https://www.destatis.de/DE/ZahlenFakten/ GesellschaftStaat/Soziales/Sozialleistungen/KinderJugendhilfe/Tabellen/ErzieherischeHilfenAusgaben2011.html.

124 St. Rspr. d. BVerfG, vgl. BVerfGE 72, 122, Ziff. 37; BVerfGE 60, 79, Ziff. 51; BVerfG ZKJ 2009, 34; BVerfG FamRZ 2010, 713; BVerfG FamRZ 2010, 528, Ziff. 28. Vgl. auch W. Roth, Die Grundrechte Minderjähriger im Spannungsfeld selbständiger Grundrechtsausübung, elterlichen Erziehungsrechts und staatlicher Grundrechtsbindung, Berlin 2003, S. 114; Jestaedt (Fn. 117), Art. 6 Abs. 2 GG Rn. 42; F. Wapler, Staatliche Reaktionsmöglichkeiten bei Kindeswohlgefährdung - verfassungsrechtliche Aspekte der jüngsten Gesetzesänderungen, RdJB 2009, S. 21 (23).

125 BVerfGE 121, 69, Ziff. 72.

126 Chr. Hohmann-Dennhardt, Kindeswohl und Elternrecht - Rechtsverhältnis von Eltern und Kindern, FÜR 2008, S. 476 f.; H.D. Jarass, in: ders./B. Pieroth, Grundgesetz, 11. Aufl. 2011, Art. 6 GG Rn. 41; von Coelln, in: M. Sachs (Hrsg.), Grundgesetz, 6. Aufl. München 2011, Art. 6 GG Rn. 54. Als „ungewöhnlich“ wird das Recht eingeordnet bei Coester-Waltjen (Fn. 47), Art. 6 GG Rn. 83, als „als solches unstreitig“ bei Brosius-Gersdorf (Fn. 47), Art. 6 GG Rn. 152. Brosius-Gersdorf postuliert im Ergebnis einen verfassungsrechtlichen Anspruch des Kindes „auf Nichtgefährdung seiner Entwicklung durch die Eltern“, den sie aus Art. 2 Abs. 1 i.V.m. Art. 1 Abs. 1 GG ableitet. Offen bleibt bei ihr die außerordentlich wichtige Frage, ob dieser Anspruch sich gegen die Eltern des Kindes oder gegen den Staat als Wächter richtet (ebd., Rn. 153).

127 BVerfG NJW 2013, 847, Ziff. 41 ff. 
überhaupt möglich machen. Diese Konstruktion fügt sich erheblich besser in die allgemeine Grundrechtsdogmatik ein, denn als Adressat des Grundrechts wird ausdrücklich der Staat genannt. Ähnlich wie beim staatlichen Wächteramt ist der Staat demnach dafür zuständig, dass Mindestbedingungen für das Kindeswohl gesichert sind bzw. dass unverzichtbare Belange des Kindes gewährleistet werden. Der Gesetzgeber ist dabei aber nicht gezwungen, im Familienrecht stets die tatsächlichen sozial-familiären Beziehungen des Kindes eins zu eins nachzuzeichnen. Beispielsweise besteht kein verfassungsrechtliches Gebot, die Sukzessivadoption in formalisierten Lebensgemeinschaften zu ermöglichen, nur weil das Kind in ihnen sozial-familiäre Beziehungen zu beiden Partnern entwickelt. Der Schutzpflicht ist nach Auffassung des Gerichts dadurch Genüge getan, dass das Kind einen rechtlichen Elternteil hat und der Partner bzw. die Partnerin ein Minimum an sorgerechtlichen Befugnissen erhält („kleines Sorgerecht“ hinsichtlich der Angelegenheiten des Alltagslebens, $\$ 1687$ b BGB, $\$ 9$ Abs. 1 LPartG). ${ }^{128}$

\section{G. Öffentliche Familienförderung}

Familie, das haben die vorstehenden Überlegungen gezeigt, wird heutzutage nicht mehr als abstrakte, vorgefundene Ordnung verstanden, sondern als eine soziale Gemeinschaft, die von den betroffenen Individuen selbst ausgestaltet wird und daher in vielfältigen Formen gelebt werden kann. Die Grundrechte der Familienmitglieder sind abstrakten Familieninteressen nicht untergeordnet, sondern begrenzen die Möglichkeiten, Familie rechtlich auszugestalten. Dies gilt auch für staatliche Interessen an der Familie, etwa für bevölkerungspolitische Zielsetzungen. ${ }^{129}$ Die unterschiedlichen Familienformen sind zudem grundsätzlich als gleichwertige Wege der Lebensgestaltung zu bewerten und vom Recht entsprechend zu behandeln. Diese verfassungsrechtlich gebotene Neutralität gegenüber unterschiedlichen familiären Lebensweisen macht die öffentliche Familienförderung zunehmend schwierig. Sind die Individuen in der Ausgestaltung ihres Familienlebens grundsätzlich frei, so muss auch die öffentliche Familienförderung die gewählten Lebensentwürfe grundsätzlich gleich behandeln. Dieses Neutralitätsgebot in der Familienförderung ist letzten Endes ein Gleichheitsrecht, mit der Konsequenz, dass Ungleichbehandlungen unterschiedlicher Familienformen einer sachlichen Rechtfertigung bedürfen. ${ }^{130}$ So klar dieser abstrakte Grundsatz formuliert ist, so widersprüchlich fallen die verfassungsrechtlichen Bewertungen der gegenwärtigen Familienleistungen aus. Als Beispiel sei nur das

128 BVerfG NJW 2013, 847, Ziff. 46. Den Ausschluss der Sukzessivadoption in Lebenspartnerschaften hat das BVerfG aber als Gleichheitsverstoß gewertet, vgl. ebd., Ziff. $71 \mathrm{ff}$.

129 Vgl. C. Seiler, Grundzüge eines öffentlichen Familienrechts, Tübingen 2008, S. 23 f.; F. BrosiusGersdorf, Demographischer Wandel und Familienförderung, Tübingen 2011, S. 194 ff.

130 Vgl. Brosius-Gersdorf, Demographischer Wandel (Fn. 128), S. 292 ff.; Seiler, Öffentliches Familienrecht (Fn. 128), S. 44 ff.; S. Huster, Die ethische Neutralität des Staates. Eine liberale Interpretation der Verfassung, Tübingen 2002, S. 533. 
Problemfeld der Kinderbetreuung genannt: Das einkommensunabhängige Elterngeld wurde als Verstoß gegen das Neutralitätsgebot kritisiert, weil es die Doppelverdienerehe bevorzuge, aber auch als neutrales und damit verfassungskonformes Instrument einer gleichheitsorientierten Familienförderung gerechtfertigt. ${ }^{131}$ Ähnlich wird das jüngst eingeführte Betreuungsgeld einerseits als Instrument gewertet, das individuelle Wahlfreiheit sichert, während die Gegenansicht darin eine verfassungswidrige Bevorzugung des traditionellen Einverdienermodells erblickt. ${ }^{132}$ Hinter diesen Kontroversen steht ein fundamentaler Konflikt zwischen dem Leitbild des ökonomisch unabhängigen und in seinen Lebensentscheidungen freien Individuums und dem Faktum, dass in jeder Gesellschaft für Kinder (und in erheblichem Maße auch für pflegebedürftige Erwachsene) gesorgt werden muss. Dieser Lebensbereich war traditionell über die familienrechtlichen Sorgepflichten der Ehefrau dem Privatleben zugeordnet und insofern kein Thema der politischen Philosophie oder des öffentlichen Rechts. Nach wie vor ist die Frage, wie Erwerbs- und Sorgepflichten in Einklang zu bringen sind, in der öffentlichen Diskussion marginalisiert. ${ }^{133}$ In diesem Sinne kann die Forderung nach einem „öffentlichen Familienrecht “ 134 nur begrüßt werden: als Wunsch nach einer politischen und verfassungsrechtlichen Diskussion über die Bedeutung fürsorglichen und solidarischen Handelns in einer politischen Gemeinschaft.

131 Verfassungsrechtliche Zweifel bei C. Seiler, Freiheits- und gleichheitsgerechte Förderung der Vereinbarkeit von Familie und Beruf, FamRZ 2010, S. 1717 (1722). Für die Verfassungsmäßigkeit K. Scheiwe/C. Fuchsloch, Rechtspolitische Ziele und rechtliche Gestaltungsmöglichkeiten eines Elterngeldes, ZRP 2006, S. 37 (39).

132 Verfassungsrechtliche Bedenken bei F. Brosius-Gersdorf, Das neue Betreuungsgeldgesetz - Familienförderung wider das Grundgesetz, NJW 2013, S. 2316 (2318 ff.); U. Sacksofsky, VerfassungsmäBigkeit des geplanten Betreuungsgeldes, Streit 2010, 167 (170). Für die Verfassungsmäßigkeit einer Aufrechnung von Leistungen der öffentlichen Infrastruktur (Kindertagesstätten u.a.) mit Direktzahlungen an Familien Seiler (Fn. 129).

133 Vgl. dazu M. Wersig, Der unsichtbare Mehrwert: Unbezahlte Arbeit und ihr Lohn, in: Lembke/Foljanty (Fn. 27), S. 173-196; aus rechtsethischer Sicht F. Wapler, Im toten Winkel der Rechtsphilosophie? Der Liberalismus und die Autonomie der Frau, ARSP-Beiheft 134, S. 79-100.

134 So Titel und Programm der Schrift von Seiler (Fn. 129). 